\title{
Detection of multiple structural breaks in multivariate time series
}

\author{
Philip Preuß, Ruprecht Puchstein, Holger Dette \\ Ruhr-Universität Bochum, Fakultät für Mathematik \\ 44780 Bochum, Germany
}

\begin{abstract}
We propose a new nonparametric procedure for the detection and estimation of multiple structural breaks in the autocovariance function of a multivariate (secondorder) piecewise stationary process, which also identifies the components of the series where the breaks occur. The new method is based on a comparison of the estimated spectral distribution on different segments of the observed time series and consists of three steps: it starts with a consistent test, which allows to prove the existence of structural breaks at a controlled type I error. Secondly, it estimates sets containing possible break points and finally these sets are reduced to identify the relevant structural breaks and corresponding components which are responsible for the changes in the autocovariance structure. In contrast to all other methods which have been proposed in the literature, our approach does not make any parametric assumptions, is not especially designed for detecting one single change point and addresses the problem of multiple structural breaks in the autocovariance function directly with no use of the binary segmentation algorithm. We prove that the new procedure detects all components and the corresponding locations where structural breaks occur with probability converging to one as the sample size increases and provide data-driven rules for
\end{abstract}


the selection of all regularization parameters. The results are illustrated by analyzing financial returns, and in a simulation study it is demonstrated that the new procedure outperforms the currently available nonparametric methods for detecting breaks in the dependency structure of multivariate time series.

Keywords and phrases: multiple structural breaks, cusum test, empirical process, nonparametric spectral estimates, multivariate time series

\section{Introduction}

The assumption of second order stationarity of time series is widely used in the statistical literature, because it yields an elegant and powerful statistical methodology like parameter estimation or forecasting techniques. On the other hand many real world phenomena can not be adequately described by stationary processes because these models are usually not able to capture all stylized facts of an observed time series $\left(\boldsymbol{X}_{\boldsymbol{t}}\right)_{t \in \mathbb{Z}}$. Consequently numerous alternative models have been proposed to address model features of non-stationarity [see Jansen et al. (1981), Dahlhaus (1997), Adak (1998) or Ombao et al. (2001) among many others], and one concept which became quite popular in the last decades is to assume that there is a certain number of segments on which the process can be considered as stationary [see for example Starica and Granger (2005) or Fryzlewicz et al. (2006)]. This implies the

existence of several points where the process 'jumps' from one stationary model to another, and testing for the presence and determining the location of such structural breaks is an important and challenging task. The main problems/questions in this context are (1) Do there exist structural breaks? (2) If there exist structural breaks, how many of them are present? (3) Where are the break points located? (4) In multivariate time series: in which components do structural breaks occur?

Because of its importance the problem of change point detection has found considerable interest in the statistical literature. For many decades, the identification of structural breaks 
in the mean function was the dominating issue, and some early results are given by Sen and Srivastava (1975). Since this pioneering work numerous authors have considered this problem [see James et al. (1987), Banerjee et al. (1992), Bai (1994) or Fryzlewicz (2007) among many others]. More recently several authors have argued that in applications the detection of changes in the autocovariance structure is of importance as well. Typical examples include the discrimination between stages of high and low asset volatility or the detection of changes in the parameters of an $\mathrm{AR}(\mathrm{p})$ model in order to obtain superior forecasting procedures. In the univariate case Inclan and Tiao (1994) propose a nonparametric CUSUM-type test for changes in the variance of an independent identically distributed sequence. These results are generalized by Lee and Park (2001) to linear processes. Chen and Gupta (1997) consider the same model and problem, but use the Schwarz information criterion. Aue et al. (2009) discuss multivariate time series and propose a nonparametric test for structural breaks in the variance matrix, which is applicable to a broad class of stochastic processes.

On the other hand, the detection of changes in the complete autocovariance structure, that is the existence of a change in the covariance function $\gamma(k)=\mathbb{E}\left(X_{0} X_{k}\right)$ at some lag $k \in \mathbb{N}$, is more complicated and the corresponding literature is much less developed and mainly refers to parametric models. Lavielle and Ludena (2000) use penalized methods for identifying structural breaks and Lee et al. (2003) propose a CUSUM-type statistic for detecting changes in specific parameters. Recently Davis et al. (2006) suggest the minimum description length principle to fit piecewise constant AR processes, while Chen et al. (2010) use AR-processes with structural breaks for modeling asset volatility.

This list is of course far from being complete and could be extended by a vast amount of further articles on this subject. Nevertheless, to the best of our knowledge, there does not exist any nonparametric method for the detection of structural breaks in the autocovariance structure of multivariate time series which addresses all four problems raised at the beginning of this paper simultaneously. In fact, essentially all procedures either assume independence of the observed data or rely on parametric assumptions. Moreover, these methods are partic- 
ularly designed to detect one structural break and the application of the binary segmentation algorithm Vostrikova (1981)] is used to locate multiple break points [see for example Fryzlewicz (2012) for a very recent approach]. Although this approach works both theoretically and practically, it is of course not necessarily optimal because it relies on procedures designed for detecting one change point.

In this paper we present an integrative procedure for the detection and localization of structural breaks in the autocovariance structure of a multivariate time series, which addresses all raised issues simultaneously. Our approach works in the frequency domain and is based on a comparison of the spectral density on two consecutive blocks of length, say $N$, which is 'small' compared to the whole sample size, say $T$. Roughly speaking, the method consists of three basic steps. We start with the development of a new consistent bootstrap test, which allows to prove the existence of structural breaks at a controlled type I error. In this case, a set of possible break points is estimated, and finally this set is reduced to identify the relevant structural breaks and corresponding components which are responsible for these breaks. The different steps are carefully described and illustrated in Sections 3 and 4 . Here we also prove that the initial test keeps its nominal level, is consistent and that the detection rule identifies all components and the corresponding locations where structural breaks occur with probability converging to one as the sample size increases.

As all methods in this context [see for example Lee et al. (2003)] the new procedure depends on the choice of several regularization parameters. Therefore we develop data-driven rules for the selection of these parameters in Section 5, which can also be justified theoretically and yield a good performance of the procedure from a practical point of view. In Section 6 we investigate the finite sample properties of the new method by means of a simulation study and illustrate its application analyzing multivariate financial market returns. In particular it is demonstrated that in most cases the new proposal improves competing nonparametric methods for detecting multiple structural breaks in the dependency structure of a multivariate time series. Finally all proofs are given in Section 7, in which some technical details are 
deferred to an additional Section 8 .

We conclude this introduction emphasizing that the focus of this paper is on nonparametric detection of changes in the autocovariance structure of multivariate time series and identification of the components which are responsible for the structural breaks. Nearly all articles on nonparametric change point detection in the dependency structure are restricted to the univariate case. To the best of our knowledge, only Aue et al. (2009) provide a formal level- $\alpha$ test for the presence of structural breaks in a multivariate time series setting. However, these authors do not investigate change point problems in the autocovariance structure, but consider the problem of testing for a constant covariance matrix. As a consequence their test is not able to discriminate between changes in the dependency structure which keeps the variance constant. This situation occurs for example in an univariate AR(1) model where the AR parameter switches from $-a$ to $a$ for some $a \in(0,1)$. The procedure proposed in this paper solves problems of this type and in the multivariate case additionally identifies the components, where the changes occur.

\section{Piecewise stationary processes}

We assume to observe realizations of a centered $\mathbb{R}^{d}$ valued stochastic process $\left(\boldsymbol{X}_{t, T}\right)_{t=1, \ldots, T}$, where $\boldsymbol{X}_{t, T}=\left(X_{t, T, 1}, \ldots, X_{t, T, d}\right)^{T}$ has a piecewise stationary representation. This means that there exist an unknown number $K \in \mathbb{N}_{0}$ and points $0=b_{0}<b_{1}<\cdots<b_{k}<b_{K+1}=1$ such that

$$
\boldsymbol{X}_{t, T}=\sum_{l=0}^{\infty} \boldsymbol{\Psi}_{l}(t / T) \boldsymbol{Z}_{t-l} \quad t=1, \ldots, T
$$

where the functions $\Psi_{l}:[0,1] \rightarrow \mathbb{R}^{d \times d}, l \in \mathbb{Z}$ are defined as $\boldsymbol{\Psi}_{l}(u)=\sum_{j=0}^{K} \Psi_{l}^{(j)} 1_{S_{j}}(u)$ and $1_{S_{j}}$ denotes the indicator function of the set $S_{j}=\left\{u: b_{j}<u \leq b_{j+1}\right\},\left\{\boldsymbol{Z}_{t}\right\}_{t \in \mathbb{Z}}$ denotes a centered Gaussian White Noise process with covariance matrix $\boldsymbol{I}_{d}$ and the matrices $\boldsymbol{\Psi}_{l}^{(j)} \in \mathbb{R}^{d \times d}$ correspond to the piecewise constant coefficents of the linear representations 
on the segment $\left(\left\lfloor b_{j} T\right\rfloor,\left\lfloor b_{j+1} T\right\rfloor\right]$. Throughout this paper we assume that $K$ is 'minimal' in the sense that for every pair $(i, i+1)$ with $i \in\{0, \ldots, K-1\}$ there exists an integer $l \in \mathbb{N}$ such that $\Psi_{l}^{(i)} \neq \Psi_{l}^{(i+1)}$. This ensures that, if $K$ equals zero, there is no change point in the dependency structure, while structural breaks exist for $K \geq 1$. We also note that the assumption of Gaussianity is only imposed here to simplify technical arguments and the extension of the proposed methodology and results to more general innovations is straigthforward; see Remark 3.9 for more details.

We introduce $\boldsymbol{f}_{j}(\lambda)=\frac{1}{2 \pi} \sum_{l, m=0}^{\infty} \Psi_{l}^{(j)}\left(\Psi_{m}^{(j)}\right)^{T} \exp (-i \lambda(l-m))$ and obtain for the $\mathbb{C}^{d \times d}$ valued time-varying (piecewise constant) spectral density matrix

$$
\boldsymbol{f}(u, \lambda)=\frac{1}{2 \pi} \sum_{l, m=0}^{\infty} \boldsymbol{\Psi}_{l}(u)\left(\boldsymbol{\Psi}_{m}(u)\right)^{T} \exp (-i \lambda(l-m))=\sum_{j=0}^{K} \boldsymbol{f}_{j}(\lambda) 1_{S_{j}}(u)
$$

From this representation it follows that the spectral density has points of discontinuity in $u$ direction at the break points $b_{i}(i=1, \ldots, K)$ whenever $K \geq 1$. Therefore we propose to compare the spectral density $\lambda \mapsto \frac{1}{e} \int_{v-e}^{v} \boldsymbol{f}(u, \lambda) d u$ with $\lambda \mapsto \frac{1}{e} \int_{v}^{v+e} \boldsymbol{f}(u, \lambda) d u$ for some 'small' constant $e$. If there exist structural breaks, the difference $\sup _{\omega \in[0,1]} \frac{1}{e} \mid \int_{0}^{\omega \pi} \int_{v}^{v+e} \boldsymbol{f}(u, \lambda) d u d \lambda-$ $\int_{0}^{\omega \pi} \int_{v-e}^{v} \boldsymbol{f}(u, \lambda) d u d \lambda \mid$ will be positive for $v \in\left\{b_{1}, \ldots, b_{K}\right\}$ while it vanishes for $v \in[0,1] \backslash\left\{b_{1}, \ldots, b_{K}\right\}$ as $e \rightarrow 0$. In order to obtain a global measure for the presence of structural breaks, we define $\|A\|_{\infty}=\max _{a, b=1, \ldots, d}\left|A_{a, b}\right|$ as the usual maximum norm of a matrix $A \in \mathbb{C}^{d \times d}$ and consider

$$
D:=\sup _{v, \omega \in[0,1]}\|\boldsymbol{D}(v, \omega)\|_{\infty}
$$

where for $v \in[e, 1-e]$ and $\omega \in[0,1]$ the matrix $\boldsymbol{D}(v, \omega)$ is defined by

$$
\boldsymbol{D}(v, \omega):=\frac{1}{e}\left(\int_{0}^{\omega \pi} \int_{v}^{v+e} \boldsymbol{f}(u, \lambda) d u d \lambda-\int_{0}^{\omega \pi} \int_{v-e}^{v} \boldsymbol{f}(u, \lambda) d u d \lambda\right) \in \mathbb{R}^{d \times d}
$$

and we set $\boldsymbol{D}(v, \omega)=\boldsymbol{D}(e, \omega)$ and $\boldsymbol{D}(v, \omega)=\boldsymbol{D}(1-e, \omega)$ whenever $v \leq e$ amd $v \geq 1-e$ respectively. Under the hypothesis of no structural break, i.e. $K=0$, we have $D=0$, while $D$ is strictly positive if structural breaks occur. In order to obtain a test for the null 
hypothesis

$$
H_{0}: \quad K=0
$$

it is therefore natural to estimate $D$ and to reject the null hypothesis for 'large' values of the estimator. We will construct such an empirical version of $D$ in the following section and derive its asymptotic properties. The identification of the location and components corresponding to the break points is based on an estimator of the components $\sup _{\omega \in[0,1]}\left|[\boldsymbol{D}(v, \omega)]_{a, b}\right|$ and illustrated in Section 4 .

\section{Testing for structural breaks}

The first step of the proposed procedure consists in a statistical test which allows to prove the existence of structural breaks at a controlled type I error and is based on an empirical version of the quantity $D$ defined in 2.3 . To be precise we choose an even integer $N \leq T / 2$ and consider the local periodogram

$$
\boldsymbol{I}_{N}(u, \lambda):=\frac{1}{2 \pi N} \sum_{r, s=0}^{N-1} \boldsymbol{X}_{\lfloor u T\rfloor-N / 2+1+s, T} \boldsymbol{X}_{\lfloor u T\rfloor-N / 2+1+r, T}^{T} \exp (-i \lambda(s-r)),
$$

where we use the convention $\boldsymbol{X}_{j, T}=0$ whenever $j \notin\{1, \ldots, T\}$. Note that 3.1 is the usual periodogram computed from the $N$ observations $\boldsymbol{X}_{\lfloor u T\rfloor-N / 2+1, T}, \ldots, \boldsymbol{X}_{\lfloor u T\rfloor+N / 2, T}$, and it can be shown that the quantity $\boldsymbol{I}_{N}(u, \lambda)$ is an asymptotically unbiased estimator for the spectral density if $N \rightarrow \infty$ [see Dahlhaus (1997)]. An estimator of the matrix $\boldsymbol{D}(v, \omega)$ is then defined by

$$
\hat{\boldsymbol{D}}_{T}(v, \omega):=\frac{1}{N} \sum_{k=1}^{\lfloor\omega N / 2\rfloor}\left(\boldsymbol{I}_{N}\left(v+N /(2 T), \lambda_{k}\right)-\boldsymbol{I}_{N}\left(v-N /(2 T), \lambda_{k}\right)\right),
$$

if $v \in\left[\frac{N}{T}, 1-\frac{N}{T}\right]$ where $\lambda_{k}=2 \pi k / N$ denote the Fourier frequencies. On the intervals $\left[0, \frac{N}{T}\right)$ and $\left(1-\frac{N}{T}, 1\right]$ we define $\hat{\boldsymbol{D}}_{T}(v, \omega)$ as $\hat{\boldsymbol{D}}_{T}\left(\frac{N}{T}, \omega\right)$ and $\hat{\boldsymbol{D}}_{T}\left(1-\frac{N}{T}, \omega\right)$ respectively. So roughly speaking we construct an estimator of $\boldsymbol{D}(v, \omega)$ by replacing the integral by a Riemann sum, where the averaged time varying spectral density matrices $\frac{1}{e} \int_{v}^{v+e} \boldsymbol{f}(u, \lambda) d u$ and 
$\frac{1}{e} \int_{v-e}^{v} \boldsymbol{f}(u, \lambda) d u$ on the intervals $[v, v+e]$ and $[v-e, v]$ are replaced by the local periodograms $\boldsymbol{I}_{N}(v+N /(2 T), \lambda)$ and $\boldsymbol{I}_{N}(v-N /(2 T), \lambda)$. The final estimate of the quantity $D$ in 2.3$)$ is then defined by

$$
\hat{D}_{T}:=\sup _{(v, \omega) \in[0,1]^{2}}\left\|\hat{\boldsymbol{D}}_{T}(v, \omega)\right\|_{\infty}=\max _{v \in[N / T, 1-N / T]} \sup _{\omega \in[0,1]}\left\|\hat{\boldsymbol{D}}_{T}(v, \omega)\right\|_{\infty} .
$$

The following results specify the asymptotic properties of the process $\left\{\hat{\boldsymbol{D}}_{T}(v, \omega)\right\}_{(v, \omega) \in[0,1]^{2}}$ under the null hypothesis 2.5 of no structural breaks and the alternative $H_{1}: K>0$ for different choices of the sequence $N$. Throughout this paper the symbol $\Rightarrow$ denotes weak convergence in $L^{\infty}\left([0,1]^{2}\right)$ and we distinguish the cases $N / T \rightarrow 1 / c \in(0,1 / 2)$ (Theorem 3.1) and $N / T \rightarrow 0$ (Theorem 3.2).

Theorem 3.1 Suppose that the coefficients in the representation (2.1) satisfy

$$
\sum_{l=0}^{\infty} \sup _{u \in[0,1]}\left\|\Psi_{l}(u)\right\|_{\infty}|l|<\infty,
$$

and that $N / T \rightarrow 1 / c$ for some $c \geq 2 / \min _{i=1, \ldots, K+1}\left|b_{i}-b_{i+1}\right|$ as $T \rightarrow \infty$. Then the following statements hold:

a) If $K=0$, the process $\left\{\sqrt{N} \hat{\boldsymbol{D}}_{T}(v, \omega)\right\}_{(v, \omega) \in[0,1]^{2}}$ converges weakly to a centered Gaussian process $\{\boldsymbol{G}(v, \omega)\}_{(v, \omega) \in[0,1]^{2}}$, i.e.

$$
\left\{\sqrt{N} \hat{\boldsymbol{D}}_{T}(v, \omega)\right\}_{(v, \omega) \in[0,1]^{2}} \Rightarrow\{\boldsymbol{G}(v, \omega)\}_{(v, \omega) \in[0,1]^{2}} .
$$

Here for $v_{i} \in\left[\frac{1}{c}, 1-\frac{1}{c}\right](i=1,2)$, the covariance kernel $\operatorname{Cov}\left(\left[\boldsymbol{G}\left(v_{1}, \omega_{1}\right)\right]_{a_{1}, b_{1}},\left[\boldsymbol{G}\left(v_{2}, \omega_{2}\right)\right]_{a_{2}, b_{2}}\right)$ of $\{\boldsymbol{G}(v, \omega)\}_{(v, \omega) \in[0,1]^{2}}$ is given by

$$
\begin{cases}0 & \text { if } \quad \frac{2}{c} \leq\left|v_{2}-v_{1}\right| \\ -\left[2-\left|v_{2}-v_{1}\right| c\right] \frac{1}{\pi} \int_{0}^{\min \left(\omega_{1}, \omega_{2}\right) \pi} \rho_{a_{1}, a_{2}, b_{1}, b_{2}}(\lambda) d \lambda & \text { if } \quad \frac{1}{c} \leq\left|v_{2}-v_{1}\right| \leq \frac{2}{c}, \\ {\left[2-3\left|v_{2}-v_{1}\right| c\right] \frac{1}{\pi} \int_{0}^{\min \left(\omega_{1}, \omega_{2}\right) \pi} \rho_{a_{1}, a_{2}, b_{1}, b_{2}}(\lambda) d \lambda} & \text { if } \quad 0 \leq\left|v_{2}-v_{1}\right| \leq \frac{1}{c}\end{cases}
$$

where $\rho_{a_{1}, a_{2}, b_{1}, b_{2}}(\lambda):=f_{a_{1}, a_{2}}(\lambda) f_{b_{1}, b_{2}}(-\lambda)+f_{a_{1}, b_{2}}(\lambda) f_{b_{1}, a_{2}}(-\lambda)$. If $v_{i} \notin\left[\frac{1}{c}, 1-\frac{1}{c}\right]$ for at 
least one $i \in\{1,2\}$ the covariance kernel is given by

$$
\operatorname{Cov}\left(\left[\boldsymbol{G}\left(v_{1}, \omega_{1}\right)\right]_{a_{1}, b_{1}},\left[\boldsymbol{G}\left(v_{2}, \omega_{2}\right)\right]_{a_{2}, b_{2}}\right)=\operatorname{Cov}\left(\left[\boldsymbol{G}\left(a_{c}\left(v_{1}\right), \omega_{1}\right)\right]_{a_{1}, b_{1}},\left[\boldsymbol{G}\left(a_{c}\left(v_{2}\right), \omega_{2}\right)\right]_{a_{2}, b_{2}}\right)
$$

where $a_{c}(v):=\min \left(\max \left(v, \frac{1}{c}\right), 1-\frac{1}{c}\right)$.

b) If $K \geq 1$, there exists a constant $C \in \mathbb{R}^{+}$with $\lim _{T \rightarrow \infty} \mathbb{P}\left(\sup _{\omega \in[0,1]}\left|\left[\hat{\boldsymbol{D}}_{T}\left(b_{r}, \omega\right)\right]_{a, b}\right|>C\right)=1$ for all $(r, a, b) \in\{1, \ldots, K\} \times\{1, \ldots, d\}^{2}$ such that $\sup _{\omega \in[0,1]}\left|\left[\boldsymbol{D}\left(b_{r}, \omega\right)\right]_{a, b}\right|>0$.

Theorem 3.2 Suppose that the coefficients in the representation (2.1) satisfy (3.4) and that $N \rightarrow \infty, T^{\varepsilon} / N \rightarrow 0$ and $N / T \rightarrow 0$ for some $\varepsilon>0$ as $T \rightarrow \infty$. Then the following statements are correct:

a) If $K=0$, then we have for any $0<\gamma<\frac{1}{2}$ : $\hat{D}_{T}=o_{P}\left(N^{-\gamma}\right)$.

b) If $K \geq 1$, then part b) of Theorem 3.1 holds.

From these results it follows that under the null hypothesis of no structural breaks the expression $\hat{D}_{T}$ is of order $O_{P}\left(N^{-\gamma}\right)$ where $\gamma=1 / 2$ (Theorem 3.1) or $\gamma \in(0,1 / 2)$ (Theorem 3.2) while it is larger than some positive constant under the alternative. Note that in the situation of Theorem 3.2 , i.e. $N / T \rightarrow 0$, it can also be shown that under the null hypothesis of no structural breaks the random variable $\sqrt{N} \hat{\boldsymbol{D}}_{T}(v, \omega)$ converges weakly to a Gaussian limit for any fixed pair $(v, \omega)$. However, in this case $\sqrt{N} \hat{\boldsymbol{D}}_{T}\left(v_{1}, \omega\right)$ and $\sqrt{N} \hat{\boldsymbol{D}}_{T}\left(v_{2}, \omega\right)$ are asymptotically uncorrelated whenever $v_{1} \neq v_{2}$, and therefore measurability is not given in the limit.

Since the distribution under the null hypothesis depends on unknown quantities of the data generating process we propose resampling methods to obtain its quantiles. More precisely we will now develop a bootstrap procedure, which is closely related to the one dimensional $\mathrm{AR}(\infty)$ bootstrap introduced by Kreiss (1988). This methodology has found considerable attention in the recent literature [see Choi and Hall (2000), Goncalves and Kilian (2007) or Berg et al. (2010) among others] since it is easy to implement but has sufficient complexity 
to capture the predominant dependencies in the underlying process. In the present context it will yield critical values such that a test for structural breaks based on the statistic $\hat{D}_{T}$ is directly implementable. For a description and the statement of the theoretical properties of the resampling procedure we require the following central assumption.

Assumption 3.3 The stationary $\mathbb{R}^{d}$-valued process $\left\{\boldsymbol{X}_{t}\right\}_{t \in \mathbb{Z}}$ with spectral density function $\boldsymbol{g}(\lambda)=\int_{0}^{1} \boldsymbol{f}(u, \lambda) d u$ has an $A R(\infty)$-representation of the form

$$
\boldsymbol{X}_{t}=\sum_{j=1}^{\infty} \boldsymbol{a}_{j} \boldsymbol{X}_{t-j}+\boldsymbol{\Sigma}^{1 / 2} \boldsymbol{Z}_{t}
$$

where $\left\{\boldsymbol{Z}_{t}\right\}_{t \in \mathbb{Z}}$ denotes a sequence of independent d-dimensional $\mathcal{N}\left(0, \boldsymbol{I}_{d}\right)$ distributed random variables, $\boldsymbol{\Sigma} \in \mathbb{R}^{d \times d}$ is positive definite and $\left(\boldsymbol{a}_{j}\right)_{j \in \mathbb{N}}$ is a sequence of $d \times d$ matrices satisfying

$$
\operatorname{det}\left(\boldsymbol{I}_{d}-\sum_{j=1}^{\infty} z^{j} \boldsymbol{a}_{j}\right) \neq 0 \text { for }|z| \leq 1 \quad \text { and } \quad \sum_{j=0}^{\infty}|j|\left\|\boldsymbol{a}_{j}\right\|_{\infty}<\infty \text {. }
$$

The main motivation of the resampling procedure consists in the fact, that every stationary process can be approximated by an $\mathrm{AR}(p)$ model if the order $p$ of the autoregressive process is sufficiently large. Therefore we choose an increasing sequence $p=p(T) \rightarrow \infty$ as $T \rightarrow \infty$ and approximate the process defined in 3.7 by an $\operatorname{AR}(p)$ model with coefficents

$$
\left(\boldsymbol{a}_{1, p}, \ldots, \boldsymbol{a}_{p, p}\right):=\underset{\boldsymbol{b}_{1, p}, \ldots, \boldsymbol{b}_{p, p}}{\operatorname{argmin}} \operatorname{tr}\left(\mathbb{E}\left[\left(\boldsymbol{X}_{t}-\sum_{j=1}^{p} \boldsymbol{b}_{j, p} \boldsymbol{X}_{t-j}\right)\left(\boldsymbol{X}_{t}-\sum_{j=1}^{p} \boldsymbol{b}_{j, p} \boldsymbol{X}_{t-j}\right)^{T}\right]\right)
$$

and innovations with covariance matrix $\boldsymbol{\Sigma}_{p}=\mathbb{E}\left[\left(\boldsymbol{X}_{t}-\sum_{j=1}^{p} \boldsymbol{a}_{j, p} \boldsymbol{X}_{t-j}\right)\left(\boldsymbol{X}_{t}-\sum_{j=1}^{p} \boldsymbol{a}_{j, p} \boldsymbol{X}_{t-j}\right)^{T}\right]$. To be precise let $\left(\hat{\boldsymbol{a}}_{1, p}, \ldots, \hat{\boldsymbol{a}}_{p, p}\right)$ denote a 'consistent' estimator of the minimizer in (3.8) (the precise assumptions regarding this estimator are specified in Theorems 3.5 and 3.6 below and are, for example, fulfilled for the Yule Walker estimators). The bootstrap replicates $\hat{D}_{T}^{*}$ of $\hat{D}_{T}$ are then generated as follows:

Algorithm 3.4 (autoregressive bootstrap) We simulate data from the model 


$$
\boldsymbol{X}_{t, T}^{*}=\sum_{j=1}^{p} \hat{\boldsymbol{a}}_{j, p} \boldsymbol{X}_{t-j, T}^{*}+\hat{\Sigma}_{p}^{1 / 2} \boldsymbol{Z}_{j}^{*}
$$

where $\hat{\boldsymbol{\Sigma}}_{p}=\frac{1}{T-p} \sum_{j=p+1}^{T}\left(\hat{\boldsymbol{z}}_{j}-\overline{\boldsymbol{z}}_{T}\right)\left(\hat{\boldsymbol{z}}_{j}-\overline{\boldsymbol{z}}_{T}\right)^{T}$, the random variables $\boldsymbol{Z}_{j}^{*}$ are independent $\mathcal{N}\left(0, \mathbf{I}_{d}\right)$ distributed, $\hat{\boldsymbol{z}}_{j}:=\boldsymbol{X}_{j, T}-\sum_{i=1}^{p} \hat{\boldsymbol{a}}_{i, p} \boldsymbol{X}_{j-i, T}(j=p+1, \ldots, T)$ and $\overline{\boldsymbol{z}}_{T}:=\frac{1}{T-p} \sum_{j=p+1}^{T} \hat{\boldsymbol{z}}_{j}$. The bootstrap statistic $\hat{D}_{T}^{*}$ is defined as the statistic $\hat{D}_{T}$ in $(3.3)$, where the observations $\left\{\boldsymbol{X}_{t, T}\right\}_{t=1, \ldots, T}$ are replaced by its bootstrap replicates $\left\{\boldsymbol{X}_{t, T}^{*}\right\}_{t=1, \ldots, T}$.

To motivate this procedure note that under the null hypothesis of stationarity the process $\left\{\boldsymbol{X}_{t}\right\}_{t=1, \ldots, T}$ and $\left\{\boldsymbol{X}_{t, T}\right\}_{t=1, \ldots, T}$ have the same spectral density $\boldsymbol{g}(\lambda)=\int_{0}^{1} \boldsymbol{f}(u, \lambda) d u=\boldsymbol{f}(\lambda)$. Now $\left\{\boldsymbol{X}_{t}\right\}_{t=1, \ldots, T}$ is approximated by an $\operatorname{AR}(p)$ process and $\left\{\boldsymbol{X}_{t, T}^{*}\right\}_{t=1, \ldots, T}$ mimics this approximation, which follows from definition (3.8) and the consistency of the estimators $\hat{\boldsymbol{a}}_{j, p}$. Therefore the process $\left\{\boldsymbol{X}_{t, T}^{*}\right\}_{t=1, \ldots, T}$ exhibits similar spectral properties as the stationary process $\left\{\boldsymbol{X}_{t}\right\}_{t=1, \ldots, T}$. Consequently, under the null hypothesis of stationarity, the distribution of $\hat{D}_{T}^{*}$ is 'close' to the distribution of the random variable $\hat{D}_{T}$. On the other hand, under the alternative, $\hat{D}_{T}^{*}$ corresponds to a stationary process with spectral density $g$, which by Theorems 3.1 and 3.2 implies $\hat{D}_{T}^{*}=O_{P}\left(N^{-\gamma}\right)$ for any $\gamma \in(0,1 / 2)$ while $\hat{D}_{T}$ becomes larger than some positive constant, which implies consistency of a bootstrap test. These heuristic arguments are made rigorous by the following statements.

Theorem 3.5 Let the assumptions of Theorem 3.1 and Assumption 3.3 be fulfilled. Furthermore, suppose that the following conditions on the growth rate of $p=p(T)$, the estimates $\hat{\boldsymbol{a}}_{j, p}$ and the true $A R$ parameters $\boldsymbol{a}_{j}, \boldsymbol{a}_{j, p}$ defined in (3.7) and (3.8) are satisfied:

i) There exist sequences $p_{\min }(T)$ and $p_{\max }(T)$ such that the order $p$ of the fitted autoregressive process satisfies $p=p(T) \in\left[p_{\min }(T), p_{\max }(T)\right]$ with $p_{\max }(T) \geq p_{\min }(T) \rightarrow \infty$ and $p_{\max }^{3}(T) \sqrt{\log (T) / T}=O(1)$.

ii) The estimators $\hat{\boldsymbol{a}}_{j, p}$ satisfy $\max _{1 \leq j \leq p(T)}\left\|\hat{\boldsymbol{a}}_{j, p}-\boldsymbol{a}_{j, p}\right\|_{\infty}=O_{P}(\sqrt{\log (T) / T})$. uniformly with respect to $p \in\left[p_{\min }(T), p_{\max }(T)\right]$. 
iii) The matrices $\hat{\Sigma}_{p}$ and $\Sigma$ satisfy $\left\|\hat{\boldsymbol{\Sigma}}_{p}-\boldsymbol{\Sigma}\right\|_{\infty} \stackrel{P}{\longrightarrow} 0$.

Then, as $T \rightarrow \infty$, we have $\left\{\sqrt{N} \hat{\boldsymbol{D}}_{T}^{*}(v, \omega)\right\}_{(v, \omega) \in[0,1]^{2}} \Rightarrow\{\boldsymbol{G}(v, \omega)\}_{(v, \omega) \in[0,1]^{2}}$ conditionally on $X_{1, T}, \ldots, X_{T, T}$, where $\boldsymbol{G}(v, \omega)$ is the limiting Gaussian process introduced in Theorem 3.1.

Theorem 3.6 Let the assumptions of Theorem 3.5 be fulfilled where (i) is replaced by $p_{\max }^{2}(T) \sqrt{\log (T) / T} N^{1+\varepsilon}=o(1)$ and $N^{1+\varepsilon} / p_{\min }(T)=o(1)$. Then there exists a sequence of $\mathbb{C}^{d \times d}$ valued random processes $\left\{\hat{\boldsymbol{D}}_{T, a}^{*}(v, \omega)\right\}_{(v, \omega) \in[0,1]}$ such that the following statements hold:

a) If $K=0$ then for any $T \in \mathbb{N}: \sup _{(v, \omega) \in[0,1]^{2}}\left\|\hat{\boldsymbol{D}}_{T}(v, \omega)\right\|_{\infty} \stackrel{D}{=} \sup _{(v, \omega) \in[0,1]^{2}}\left\|\hat{\boldsymbol{D}}_{T, a}^{*}(v, \omega)\right\|_{\infty}$.

b) If $K \geq 0$, then (as $T \rightarrow \infty$ )

$$
\frac{\sup _{(v, \omega) \in[0,1]^{2}}\left\|\hat{\boldsymbol{D}}_{T}^{*}(v, \omega)\right\|_{\infty}-\sup _{(v, \omega) \in[0,1]^{2}}\left\|\hat{\boldsymbol{D}}_{T, a}^{*}(v, \omega)\right\|_{\infty}}{\mathbb{E}\left(\sup _{(v, \omega) \in[0,1]^{2}}\left\|\hat{\boldsymbol{D}}_{T, a}^{*}(v, \omega)^{2}\right\|_{\infty}\right)^{-1 / 2}}=o_{P}(1)
$$

c) For any $0<\gamma<\frac{1}{2}: N^{\gamma} \sup _{(v, \omega) \in[0,1]^{2}}\left\|\hat{\boldsymbol{D}}_{T, a}^{*}(v, \omega)\right\|_{\infty}=o_{P}(1)$.

Note that all assumptions of Theorems 3.5 and 3.6 are rather standard in the framework of $\operatorname{AR}(\infty)$ bootstrap [see for example Berg et al. (2010) or Kreiss et al. (2011) among others] and that (ii) is proved in Hannan and Kavalieris (1986) for the least squares and the YuleWalker estimators. In order to obtain a consistent level $\alpha$ test for the null hypothesis (2.5), we now proceed as follows:

Step I (testing for structural breaks) We calculate the test statistic $\hat{D}_{T}$ defined in 3.3 and fit an $\operatorname{AR}(p)$ model to the observed data $\left\{\boldsymbol{X}_{1, T}, \ldots, \boldsymbol{X}_{T, T}\right\}$. In the next step Algorithm 3.4 with the corresponding estimates $\left(\hat{\boldsymbol{a}}_{1, p}, \ldots, \hat{\boldsymbol{a}}_{p, p}, \hat{\boldsymbol{\Sigma}}_{p}\right)$ is used to calculate the bootstrap sample $\left\{\hat{D}_{T, 1}^{*}, \ldots, \hat{D}_{T, B}^{*}\right\}$ and the null hypothesis of no break points is rejected, whenever

$$
\hat{D}_{T}>\hat{D}_{T,(\lfloor(1-\alpha)\rfloor B)}^{*},
$$

where $\hat{D}_{T,(\lfloor(1-\alpha)\rfloor B)}^{*}$ is an estimate for the $(1-\alpha)$-quantile of the distribution of $\hat{D}_{T}$ (here $\hat{D}_{T,(1)}^{*}, \ldots, \hat{D}_{T,(B)}^{*}$ denotes the ordered bootstrap sample). 
Theorem 3.5 and the continuous mapping theorem imply that the test constructed in (3.11) has asymptotic level $\alpha$ under the assumption $N / T \rightarrow 1 / c$. On the other hand in the case $N / T \rightarrow 0$ it can be show by application of Theorem 3.6 that the Mallows distance between the random variables

$$
\begin{aligned}
& \sup _{(v, \omega) \in[0,1]^{2}}\left\|\hat{\boldsymbol{D}}_{T}(v, \omega)\right\|_{\infty} / \sqrt{\mathbb{E} \sup _{(v, \omega) \in[0,1]^{2}}\left\|\hat{\boldsymbol{D}}_{T}(v, \omega)^{2}\right\|_{\infty},} \\
& \sup _{(v, \omega) \in[0,1]^{2}}\left\|\hat{\boldsymbol{D}}_{T}^{*}(v, \omega)\right\|_{\infty} / \sqrt{\mathbb{E} \sup _{(v, \omega) \in[0,1]^{2}}\left\|\hat{\boldsymbol{D}}_{T}^{*}(v, \omega)^{2}\right\|_{\infty}}
\end{aligned}
$$

converges to zero in probability. Therefore similar arguments as in Paparoditis (2010) indicate that the bootstrap test has asymptotic level $\alpha$ if $N / T \rightarrow 0$. Moreover, the test is consistent under the alternative in both cases, since it follows from Theorem 3.5 and 3.6 that the bootstrap statistic $\sup _{(v, \omega) \in[0,1]^{2}}\left\|\hat{\boldsymbol{D}}_{T}^{*}(v, \omega)\right\|_{\infty}$ converges to zero in probability, while $\sup _{(v, \omega) \in[0,1]^{2}}\left\|\hat{\boldsymbol{D}}_{T}(v, \omega)\right\|_{\infty}$ becomes larger than some positive constant under the alternative due to Theorem 3.1 b) and $3.2 \mathrm{~b}$ ). The finite sample properties of this test will be investigated in Section 6 .

Remark 3.7 If the statistic in $(3.3)$ is replaced by $\sup _{v \in[0,1]}\left\|\hat{\boldsymbol{D}}_{T}(v, 1)\right\|_{\infty}$, a similar analysis can be performed and we obtain as a special case a test for the null hypothesis that the covariance matrix of the process is constant over time as considered in Aue et al. (2009). Moreover, several other interesting hypotheses can be included by choosing an appropriate function $\phi(u, \lambda):[0,1] \times[0, \pi] \rightarrow \mathbb{C}^{d \times d}$ and considering functionals of the form

$$
\frac{1}{e}\left(\int_{0}^{\omega \pi} \int_{v}^{v+e} \boldsymbol{\phi}(u, \lambda) \boldsymbol{f}(u, \lambda) d u d \lambda-\int_{0}^{\omega \pi} \int_{v-e}^{v} \boldsymbol{\phi}(u, \lambda) \boldsymbol{f}(u, \lambda) d u d \lambda\right)
$$

instead of $\boldsymbol{D}(v, \omega)$. For example, the choice $\boldsymbol{\phi}(u, \lambda)=\boldsymbol{I}_{d} \exp (-i \lambda k)$ yields a test for constancy of the covariance function at a specific lag $k \in \mathbb{N}$. A hypothesis of this type is of interest if the statistician knows in advance that only certain lags influence the dependency structure of the underlying process. 
Remark 3.8 Statistics with a similar structure as $\hat{\boldsymbol{D}}_{T}(v, \omega)$ have been studied under different conditions by several authors in the past and some references can be found in Giraitis and Leipus (1990). These authors consider one dimensional time series and the periodogram $I_{\left(k_{1}, \ldots, k_{2}\right)}(\lambda)$ of the data $X_{k_{1}}, X_{k_{1}+1}, \ldots, X_{k_{2}}$. In order to test for a change point they propose

to compare the estimators $\hat{F}_{k}(\omega)=\int_{0}^{\omega \pi} I_{(1, \ldots, k)}(\lambda)$ and $\hat{F}_{T-k}^{*}(\omega)=\int_{0}^{\pi \omega} I_{(k+1, \ldots, T)}(\lambda) d \lambda$ by calculating $\sup _{k \in\{1, \ldots, T\}} \sup _{\omega \in[0,1]}\left|\hat{F}_{k}(\omega)-\hat{F}_{T-k}^{*}(\omega)\right|$. Note that this procedure can detect at most one break point and that these authors show that an appropriately standardized test statistic converges to some non degenerate limit whose quantiles are, however, unknown. These results are of great importance to understand the theoretical properties of such statistics. On the other hand, the construction of a computable level $\alpha$ test based on statistics of this type is an open and very challenging problem.

Remark 3.9 We emphasize that the assumption of Gaussianity in this (and the following) section is merely imposed to simplify technical arguments in the proofs. In particular it is straightforward (but cumbersome) to extend the results to a more general class of linear processes. In fact, the proof of Theorem 3.1 can be modified to address for non Gaussian innovations. The only difference appears in the bootstrap procedure because Gaussian innovations are not an appropriate choice of the bootstrap replicates $\boldsymbol{Z}_{t}^{*}$ in Algorithm 3.4 . In general one has to employ replicates $\boldsymbol{Z}_{t}^{*}$ which mimic the fourth cumulant of the true underlying innovation process [see Kreiss and Paparoditis (2012) for more details].

\section{Detecting the number and location of break points}

If structural breaks have been detected by the test (3.11) it is of futher interest to estimate the number and location of possible break points and to identify the components responsible for these changes in the regime. In the following discussion we will develop a procedure which consists of two further steps and detects simultaneously the number, location and corresponding components of multiple structural breaks. In the second step we estimate 
(shrinking if $N / T \rightarrow 0$ ) sets, which may contain potential break points. Roughly speaking these sets contain all points where the components of the spectral density estimate indicate a structural break. In the third step these sets are reduced to identify the relevant structural breaks and corresponding components which are responsible for these breaks. For this purpose we recall the definition $(3.2)$, choose some constant $0<\gamma<1 / 2$ (a recommendation for this choice will be given in Section 5) and proceed as follows.

Step II (identification of sets containing break points) We consider a point $v \in$ $\left\{\frac{N}{T}, \frac{N+1}{T}, \ldots, \frac{T-N}{T}\right\}$ as a candidate for a structural break in the component $(a, b)$ if the inequality

$$
N^{\gamma} \sup _{\omega \in[0,1]}\left|\left[\hat{\boldsymbol{D}}_{T}(v, \omega)\right]_{a, b}\right|>\boldsymbol{\varepsilon}_{T, a, b}(v)
$$

holds, where $\varepsilon_{T, a, b}(v)$ is a threshold satisfying $\liminf _{T \rightarrow \infty} \varepsilon_{T, a, b}(v) \geq C>0$ for some constant $C$ and $\varepsilon_{T, a, b}(v)=o\left(N^{\gamma}\right)$ uniformly in $v \in[0,1]$. A data driven rule for the choice of the threshold $\varepsilon_{T, a, b}(v)$ with good finite sample properties will be given in Section 5 .

The decision rule (4.1) identifies subsets $R_{1}, \ldots, R_{K_{T}} \subset\{N / T, \ldots, 1-N / T\}$ where possible break points in the components of the spectral density matrix may occur. The following example illustrates the second step of the procedure.

Example 4.1 We consider the bivariate model

$$
\boldsymbol{X}_{t, T}=\sum_{j=1}^{4} 1_{\left(\frac{j-1}{4} T, \frac{j}{4} T\right]}(t) \Theta_{j} \boldsymbol{Z}_{t}
$$

where the matrices $\Theta_{1}, \Theta_{2}, \Theta_{3}, \Theta_{4}$ are defined by

$$
\Theta_{1}:=\left(\begin{array}{ll}
1 & 0 \\
0 & 1
\end{array}\right) \quad \Theta_{2}:=\left(\begin{array}{ll}
2 & 0 \\
0 & 1
\end{array}\right) \quad \Theta_{3}:=\left(\begin{array}{ll}
2 & 0 \\
0 & 2
\end{array}\right) \quad \Theta_{4}:=\left(\begin{array}{cc}
\sqrt{2} & \sqrt{2} \\
0 & 2
\end{array}\right)
$$

and $\left\{\boldsymbol{Z}_{t}\right\}_{t \in \mathbb{Z}}$ is a two dimensional Gaussian white noise process. The spectral density $\boldsymbol{f}$ of a bivariate time series model 4.2 exhibits 3 break points, where the first change only involves the first component $[\boldsymbol{f}]_{1,1}$, the second only concerns the component $[\boldsymbol{f}]_{2,2}$ and the third break point leaves the compontens $[\boldsymbol{f}]_{1,1}$ and $[\boldsymbol{f}]_{2,2}$ unchanged but appears in the cross 

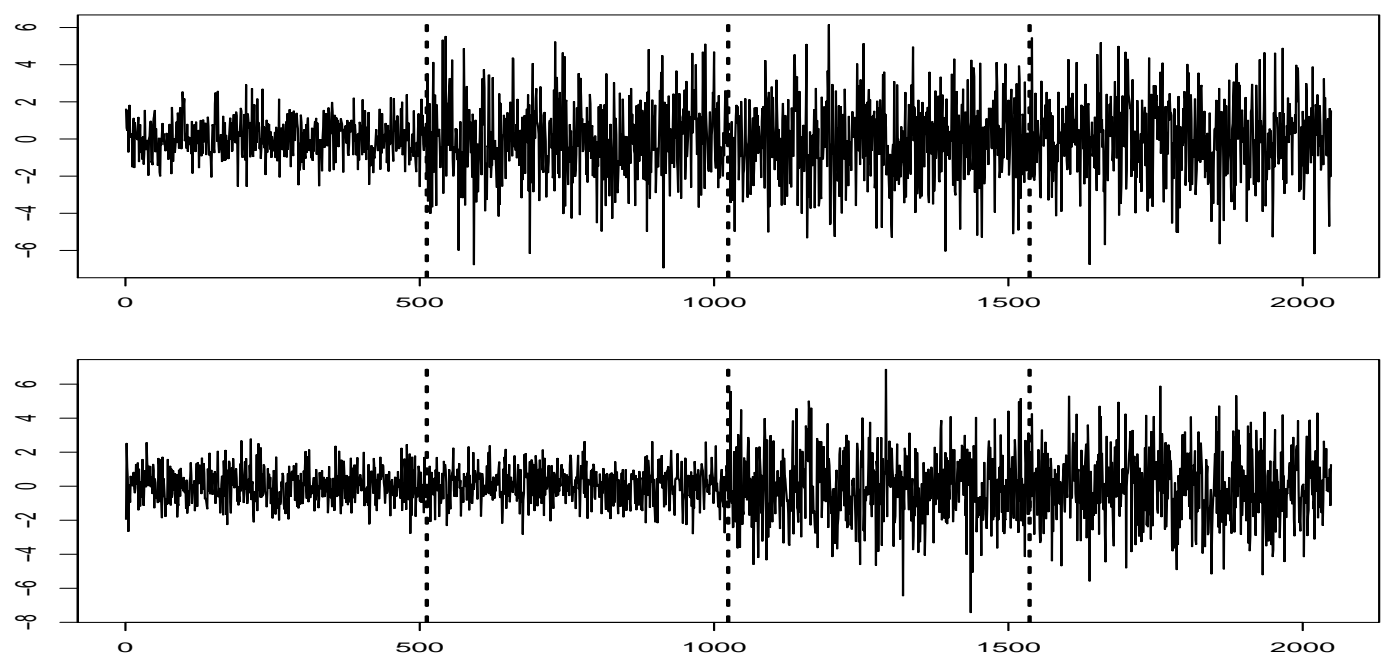

Figure 1: Simulated data from the model (4.2), where $T=2048$. The vertical dashed lines denote the true break points in the univariate time series.

spectrum $[\boldsymbol{f}]_{1,2}$ and $[\boldsymbol{f}]_{2,1}$. Figure 1 contains a plot of a typical set of data of length $T=2048$ generated by model 4.2 , where the dashed vertical lines indicate the true break points in the univariate time series. Note that the third break point only corresponds to a change in the dependency structure of the two univariate data sets. Figure 2 shows the four plots of the functions $v \mapsto N^{\gamma} \sup _{\omega \in[0,1]}\left|\left[\hat{D}_{T}(v, \omega)\right]_{a, b}\right|, a, b \in\{1,2\}$, (solid lines), where $N=256, \gamma=0.49$. In each component we added a plot of the threshold level $v \mapsto \varepsilon_{T, a, b}(v), a, b \in\{1,2\}$ (broken lines), which will be formally defined in equation (5.1) of the following section. It is evident that for each component $(a, b)$ the test statistic exceeds the level $\varepsilon_{T, a, b}(v)$ in a neighborhood of the break point. For the simulated scenario we obtain the sets $R_{1}=\left\{\frac{289}{2048}, \ldots, \frac{606}{2048}\right\}$, $R_{2}=\left\{\frac{802}{2048}, \ldots, \frac{1140}{2048}\right\}, R_{3}=\left\{\frac{1427}{2048}, \ldots, \frac{1715}{2048}\right\}$ of potential break points. Note that the local maximum of the function $N^{\gamma} \sup _{\omega \in[0,1]}\left|\left[\hat{\boldsymbol{D}}_{T}(v, \omega)\right]_{a, b}\right|$ is rather close to the true change point.

The following result shows that for an increasing sample size the subsets $R_{1}, \ldots, R_{K_{T}}$ are contained in neighborhoods of radius of $\frac{N}{T}$ of the 'true' break points, that is

$$
\bigcup_{j=1}^{K_{T}} R_{j} \subset \bigcup_{a, b \in\{1, \ldots, d\}} \mathcal{I}_{T, a, b}\left(b_{1}, \ldots, b_{K}\right),
$$



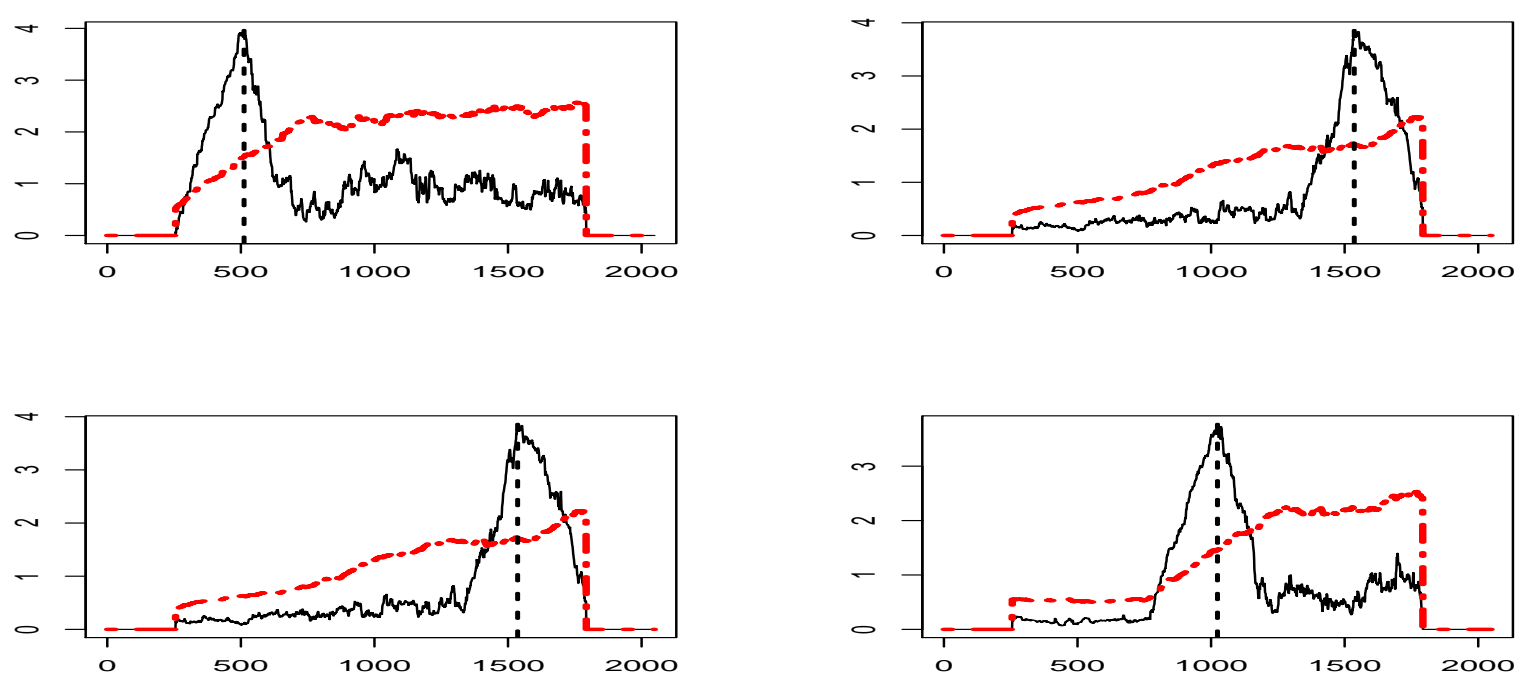

Figure 2: Plots of the functions $v \mapsto N^{\gamma} \sup _{\omega \in[0,1]}\left|\left[\hat{D}_{T}(v, \omega)\right]_{a, b}\right|$ (solid lines) and $v \mapsto \varepsilon_{T, a, b}(v)$ $(a, b=1,2)$ (broken lines) with vertical dashed lines at the true break points.

where

$$
\mathcal{I}_{T, a, b}\left(b_{1}, \ldots, b_{K}\right):=\bigcup_{\substack{j=1 \\ \sup _{\omega}\left|\left[D\left(b_{j}, \omega\right)\right]_{a, b}\right|>0}}^{K}\left\{\frac{\left\lfloor b_{j} T\right\rfloor-N}{T}, \ldots, \frac{\left\lfloor b_{j} T\right\rfloor+N}{T}\right\}
$$

Theorem 4.1 Assume that condition (3.4) is satisfied and that the sequence $N$ satisfies one of the following conditions:

(1) There exists some $\varepsilon>0$ such that $N \rightarrow \infty, T^{\varepsilon} / N \rightarrow 0$ and $N / T \rightarrow 0$.

(2) There exists some constant $c \geq 2 / \min _{i=1, \ldots, K-1}\left|b_{i}-b_{i+1}\right|$ such that $N / T \rightarrow 1 / c$.

Furthermore for all $a, b \in\{1, \ldots, d\}, v \in[0,1]$ and $\gamma \in\left(0, \frac{1}{2}\right)$ let $\left(\varepsilon_{T, a, b}(v)\right)_{T \in \mathbb{N}}$ denote a sequence satisfying $\varepsilon_{T, a, b}(v)=o\left(N^{\gamma}\right)$ and $\liminf _{T \rightarrow \infty} \inf _{v \in[0,1]} \varepsilon_{T, a, b}(v) \geq C>0$ for some constant $C$. Then the detection rule (4.1) is accurate in the following sense:

a) The probability that the decision rule (4.1) indicates a structural break at a rescaled time point, which has a distance of at least $\frac{N}{T}$ from each of the break points $b_{1}, \ldots, b_{K}$, vanishes asymptotically, that is 


$$
\mathbb{P}\left(\bigcup_{a, b \in\{1, \ldots, d\}} \bigcup_{v \in \overline{\mathcal{I}}_{T, a, b}\left(b_{1}, \ldots, b_{K}\right)}\left\{N^{\gamma} \sup _{\omega \in[0,1]}\left|\left[\hat{\boldsymbol{D}}_{T}(v, \omega)\right]_{a, b}\right|>\boldsymbol{\varepsilon}_{T, a, b}(v)\right\}\right) \stackrel{T \rightarrow \infty}{\longrightarrow} 0
$$

where $\overline{\mathcal{I}}_{T, a, b}\left(b_{1}, \ldots, b_{K}\right)=\left\{\frac{N}{T}, \frac{N+1}{T}, \ldots, \frac{T-N}{T}\right\} \backslash \mathcal{I}_{T, a, b}\left(b_{1}, \ldots, b_{K}\right)$.

b) The probability that the procedure detects all structural breaks converges to one, that is

$$
\mathbb{P}\left(\bigcap_{v \in\left\{b_{1}, \ldots, b_{K}\right\}} \bigcap_{(a, b) \in B(v)}\left\{N^{\gamma} \sup _{\omega \in[0,1]}\left|\left[\hat{\boldsymbol{D}}_{T}(v, \omega)\right]_{a, b}\right|>\boldsymbol{\varepsilon}_{T, a, b}(v)\right\}\right) \stackrel{T \rightarrow \infty}{\longrightarrow} 1,
$$

where $B(v):=\left\{(a, b) \in\{1, \ldots, d\}^{2}\left|\sup _{\omega \in[0,1]}\right|[\boldsymbol{D}(v, \omega)]_{a, b} \mid>0\right\}$.

Recall that we use the decision rule (4.1) to identify sets of possible break points. By Theorem 4.1 it follows with probability converging to 1 that these sets are contained in the (shrinking if $N / T \rightarrow 0$ ) neighborhoods $\left\{\frac{\left\lfloor b_{j} T\right\rfloor-N}{T}, \ldots, \frac{\left\lfloor b_{j} T\right\rfloor+N}{T}\right\}$ of those points $b_{j}$, for which there is at least one change in one of the components of the spectral density matrix. As demonstrated in Example 4.1 the inequality (4.1) is usually satisfied in a neighborhood of the break point. Consequently, for a finite sample size, the number of possible change points detected by (4.1) is usually much larger than the true number $K \in \mathbb{N}$. To address this issue these sets are further reduced in the third step of the procedure. As a final result we obtain estimators of the number and the location of structural breaks. The basic idea is very simple: For a certain set $R_{j}$ of points in $\{N / T, \ldots, 1-N / T\}$ satisfying (4.1) for at least one pair $(a, b) \in\{1, \ldots, d\}^{2}$ we identify a point $\tilde{b} \in R_{j}$ for which the local deviation from stationarity is maximal and then remove all points of the interval $\left[\tilde{b}-\frac{N}{T}, \tilde{b}+\frac{N}{T}\right]$ from the set $R_{j}$. The detailed description of this idea, which has to be iterated, is described below.

\section{Step III (localization of structural breaks)}

(1) Let $\hat{K}$ denote the number of elements $v \in\left\{\frac{N}{T}, \frac{N+1}{T}, \ldots, \frac{T-N}{T}\right\}$ which fulfill (4.1) for at least one component $(a, b) \in\{1, \ldots, d\}^{2}$, denote the corresponding elements by $\hat{b}_{1}, \ldots, \hat{b}_{\hat{K}}$ and define the sets $\hat{B}_{P}:=\left\{\hat{b}_{1}, \ldots, \hat{b}_{\hat{K}}\right\}$ and $\hat{B}_{D}=\emptyset$ of potential break points and detected break points, respectively.

(2) If the set $\hat{B}_{P}$ is not empty add the element $\tilde{b} \in \hat{B}_{P}$ to the set $\hat{B}_{D}$ for which the 
statistic $\sup _{(a, b) \in\{1, \ldots, d\}^{2}}\left(\sup _{\omega \in[0,1]} N^{\gamma} \mid\left[\hat{\boldsymbol{D}}_{T}(\tilde{b}, \omega)\right]_{a, b}\right)$ is maximal and replace the set $\hat{B}_{P}$ by $\hat{B}_{P} \backslash\left[\tilde{b}-\frac{N}{T}, \tilde{b}+\frac{N}{T}\right]$.

(3) Repeat step (2) until $B_{P}=\emptyset$ and redefine $\hat{K}=\left|\hat{B}_{D}\right|$ and $\left(\hat{b}_{1}, \ldots, \hat{b}_{\hat{K}}\right)$ as the elements of $\hat{B}_{D}$ such that $\hat{b}_{i}<\hat{b}_{i+1}$ for $i=1, \ldots, \hat{K}-1$.

Note that in step (2) of the above procedure we pick the element from $\hat{B}_{P}$ for which a change in the spectral density matrix seems to be most noticable. If $\hat{K}$ break points $\hat{b}_{1}, \ldots, \hat{b}_{K}$ have been found, it is (as mentioned above) of further interest to determine the nature of each detected structural break, i.e. to identify the components of the spectral density matrix, which exhibit a change at the specific points in time. Such a refined analysis can be undertaken by considering a change in the component $(a, b)$ of the spectral density matrix $\boldsymbol{f} \in \mathbb{C}^{d \times d}$ at the point $\hat{b}_{i}$ as relevant if the inequality $N^{\gamma} \sup _{\omega \in[0,1]}\left|\left[\hat{\boldsymbol{D}}_{T}\left(\hat{b}_{i}, \omega\right)\right]_{a, b}\right|>\boldsymbol{\varepsilon}_{T, a, b}\left(\hat{b}_{i}\right)$ holds.

Example 4.2 In order to illustrate this step we continue the discussion of Example 4.1 where the sets $R_{1}, R_{2}$ and $R_{3}$ of potential break points were identified. Therefore at the beginning of Step III the set of possible break points is given by $\hat{B}_{P}=R_{1} \cup R_{2} \cup R_{3}$ while the set of determined break points satisfies $\hat{B}_{D}=\emptyset$. In the first step we add the element $\tilde{b}=\frac{511}{2048}$ to $\hat{B}_{D}$ and then remove all elements, which do not have a distance of at least $N / T=\frac{256}{2048}$ from $\tilde{b}=\frac{511}{2048}$, from the set $\hat{B}_{P}$. This leaves the set $\hat{B}_{P}=R_{2} \cup R_{3}$. In the next iteration we add the element $\tilde{b}=\frac{1537}{2048}$ to $\hat{B}_{D}$ and reduce the set $\hat{B}_{P}$ to $\hat{B}_{P}=R_{2}$. In the last step we add the point $\tilde{b}=\frac{1026}{2048}$ and obtain $\hat{B}_{D}=\left\{\frac{511}{2048}, \frac{1026}{2048}, \frac{1537}{2048}\right\}$ and reduce the set $\hat{B}_{P}$ accordingly. At this point the procedure terminates because $\hat{B}_{P}=\emptyset$ and yields $\hat{K}=3$ as an estimator for the number of break points. The locations of the break points are estimated as $\left(\hat{b}_{1}, \hat{b}_{2}, \hat{b}_{3}\right)=\left(\frac{511}{2048}, \frac{1026}{2048}, \frac{1537}{2048}\right)$. Thus in this example all break points are detected and the respective components of the spectral density matrix $f$ responsible for these changes are identified as well.

Theorem 4.1 implies that $\hat{K}$ converges to $K$ in probability and that the same statement also 
holds for the estimated break points $\hat{b}_{1}, \ldots, \hat{b}_{\hat{K}}$, that is

$$
\hat{b}_{j} \stackrel{P}{\longrightarrow} b_{j} \quad(j=1, \ldots, K),
$$

where $b_{1}, \ldots, b_{K}$ denotes the true locations of the break points. In the case (1) of shrinking relative size of the blocklength $N / T \rightarrow 0$, the property 4.6 simply follows from the fact that the diameter of the sets $R_{j}$ converges to zero. For sequences $N$ which satisfy condition (2), consistency follows from the property

$$
N^{\gamma} \sup _{(v, \omega) \in[0,1]^{2}}\left\|\hat{\boldsymbol{D}}_{T}(v, \omega)-\boldsymbol{D}_{N, T}(v, \omega)\right\|_{\infty}=o_{P}(1)
$$

where $\boldsymbol{D}_{N, T}(v, \omega):=\frac{T}{N}\left(\int_{0}^{\omega \pi} \int_{v}^{v+N / T} \boldsymbol{f}(u, \lambda) d u d \lambda-\int_{0}^{\omega \pi} \int_{v-N / T}^{v} \boldsymbol{f}(u, \lambda) d u d \lambda\right)$. This can be shown by similar arguments as given in the proof of Theorem 3.1 and the fact that the deterministic sequence $\bar{D}_{N, T}(v):=\sup _{\omega \in[0,1]}\left\|\boldsymbol{D}_{N, T}(v, \omega)\right\|$ attains local maxima at the true break points $b_{j}$ as $T \rightarrow \infty$. In addition, Theorem 4.1 also shows that the refined analysis described in the previous paragraph consistently identifies the components where the structural breaks are present. This means that the procedure is able to detect the 'true' number, locations and components responsible for the breaks with a probability converging to one as the sample size increases. We will give data driven rules for the choice of the regularization parameters in Section 5 and investigate the finite sample properties of the procedure in Section 6 .

\section{Data driven regularization}

The proposed test for structural breaks and the corresponding localization method depend on the choice of several regularization parameters. In this section we will present guidelines for a data driven choice of these parameters which from a theoretical point of view satisfy the assumptions for the asymptotic theory discussed in the previous sections and from a practical point of view, yield good results for finite samples (see the examples of the following section). 
We begin with the choice of $\gamma$, where one faces a tradeoff in practical applications. Roughly speaking large values of $\gamma \in\left(0, \frac{1}{2}\right)$ tend to increase the number $\hat{K}$ of estimated break points. This property is of practical importance for example if it is desirable to avoid an underestimation of the true number of break points. In our simulation study we follow this path (i.e. we want to be sure to detect all underlying change points) and choose $\gamma=0.49$. This value leads to satisfactory results in all investigated scenarios (see Section 6 for more details) and is therefore recommended.

For the choice of the threshold $\varepsilon_{T, a, b}(v)$ in 4.1 we note that it follows by similar calculations as given in the proof of Theorem 3.1 that the variance of $\left[\hat{\boldsymbol{D}}_{T}(v, \omega)\right]_{a, b}$ satisfies

$$
\begin{aligned}
\lim _{\substack{T \rightarrow \infty \\
N / T \rightarrow c}} \operatorname{Var}\left(\sqrt{N}\left[\hat{\boldsymbol{D}}_{T}(v, \omega)\right]_{a, b}\right)=\frac{1}{2 \pi} \int_{0}^{\pi \omega}\left(\left[\boldsymbol{f}\left(v-\frac{1}{2 c}, \lambda\right)\right]_{a a}\left[\boldsymbol{f}\left(v-\frac{1}{2 c}, \lambda\right)\right]_{b b}\right. \\
\left.\quad+\left[\boldsymbol{f}\left(v+\frac{1}{2 c}, \lambda\right)\right]_{a a}\left[\boldsymbol{f}\left(v+\frac{1}{2 c}, \lambda\right)\right]_{b b}+\left|\left[\boldsymbol{f}\left(v-\frac{1}{2 c}, \lambda\right)\right]_{a b}\right|^{2}+\left|\left[\boldsymbol{f}\left(v+\frac{1}{2 c}, \lambda\right)\right]_{a b}\right|^{2}\right) d \lambda .
\end{aligned}
$$

Consequently, for fixed $v, \omega$, the asymptotic local variance can be easily estimated on a block of length $2 N$ by $M_{T, a, b}(v, \omega)=\frac{1}{N} \sum_{k=1}^{\lfloor\omega\rfloor}\left[\boldsymbol{I}_{2 N}\left(v, \lambda_{k, 2 N}\right)\right]_{a a}\left[\boldsymbol{I}_{2 N}\left(v, \lambda_{k, 2 N}\right)\right]_{b b}$, where $\lambda_{k, 2 N}:=$ $2 \pi k / 2 N$. Similar arguments as given in the proof of Theorem 3.1 yield that $N^{\gamma}\left[\hat{\boldsymbol{D}}_{T}(v, \omega)\right]_{a, b}$ and $N^{\gamma}\left[\hat{\boldsymbol{D}}_{T}\left(v^{\prime}, \omega^{\prime}\right)\right]_{a, b}$ are asymptotically independent whenever $v \neq v^{\prime}$. Motivated by hard thresholding [see Fan (1994)] we recommend

$$
\boldsymbol{\varepsilon}_{T, a, b}(v)=\sqrt{2 M_{T, a, b}(v, 1) \log \left(\frac{d(d+1) T}{2 N}\right)},
$$

where $d$ denotes the dimension of the time series under consideration. Note that under the condition $\inf _{u \in[0,1]} \min _{i \in\{1, \ldots, d\}}[\boldsymbol{f}(u, \lambda)]_{i i}>0$ it follows that $\liminf _{T \rightarrow \infty} \boldsymbol{\varepsilon}_{T, a, b}(v) \geq C>0$ for all $v \in[0,1]$, $a, b \in\{1, \ldots, d\}$ with probability converging to 1 , and that the threshold sequence (5.1) thus satisfies the conditions of Theorem 4.1.

The choice of the window length $N$ has to reflect two contradicting objectives. On the one hand, $N$ should be chosen rather large, if there exists no change point or if there are only a few structural breaks with long stationary segments in between. On the other hand, $N$ 
should be chosen rather small if there exist many break points with small distances. This basic idea is incorporated in the following rule.

Algorithm 5.1 (choice of the window length $N$ ) We consider a set of even integers, say $V_{T}:=\left\{N_{1}, \ldots, N_{n}\right\}$ satisfying $\sqrt{T} \leq N_{1}<N_{2}<\ldots<N_{n} \leq T^{5 / 6}$ and determine for each $N \in V_{T}$ the number $\hat{K}_{T}(N)$ of break points detected by the algorithm of Step III. We define $i^{*}:=\sup \left\{i \in\{2, \ldots, n(T)\} \mid \hat{K}_{T}\left(N_{i-1}\right) \leq \hat{K}_{T}\left(N_{i}\right)\right\}($ here $\sup \emptyset=-\infty), N^{*}=N_{i^{*}}$ if $i^{*} \leq n(T)$ and $N^{*}=N_{n(T)}$ if $i^{*}=-\infty$ and use $N=2 N^{*}$ for the test of structural breaks and $N=N^{*}$ for the estimation of $K$ and the localization of the break points.

Algorithm 5.1 employs the detection method described in Section 4 for every $N \in V_{T}$ and selects $N^{*}$ as the largest $N \in V_{T}$ for which there is no additional break point detected for the next smaller $N \in V_{T}$. Note also that the recommended choice of $N$ is different for step I and steps II, III of the procedure. This recommendation is motivated by the following observation. If the distance between two consecutive break points is $m$, then one should use $N=m$ in step I, while the 'best' window length would be $N=m / 2$ in step II [because we assume that the distance between two consecutive change points is at least $2 / c]$. In the numerical investigations we additionally restrict ourselves to window lengths which equal a power of two, that is we consider $n=\left\lfloor\log _{2}\left(T^{5 / 6}\right)\right\rfloor-\left\lceil\log _{2}(\sqrt{T})\right\rceil+1$ and $N_{i}=2^{\left\lceil\log _{2}(\sqrt{T})\right\rceil-1+i}$ for $i=1, \ldots, n$. This choice allows for an application of the FFT algorithm in the calculation of the local periodogram which yields a significant reduction in computational time.

Finally we select the order $p$ for the autoregressive bootstrap as the minimizer of the AIC criterion, which is defined by

$$
\hat{p}=\operatorname{argmin}_{p} \frac{2 \pi}{T} \sum_{k=1}^{T / 2}\left(\log \left(\operatorname{det}\left[\boldsymbol{f}_{\hat{\theta}(p)}\left(\lambda_{k, T}\right)\right]\right)+\operatorname{tr}\left[\left(\boldsymbol{f}_{\hat{\theta}(p)}\left(\lambda_{k, T}\right)\right)^{-1} \boldsymbol{I}_{T}\left(\lambda_{k, T}\right)\right]\right)+p / T
$$

in the context of stationary processes [see Whittle (1951)]. Here, $\boldsymbol{f}_{\hat{\theta}(p)}$ is the spectral density of the fitted stationary $\operatorname{AR}(p)$ process and $\boldsymbol{I}_{T}$ is the usual periodogram calculated under the assumption of stationarity with the corresponding Fourier frequencies $\lambda_{k, T}=2 \pi k / T$. 


\section{$6 \quad$ Finite sample properties}

In this section we investigate the finite sample properties of the proposed procedure. We also provide a comparison with competing methods and illustrate the methodology in a data example. The regularizing parameters are chosen as described in the previous section. Throughout this paragraph $\left\{\boldsymbol{Z}_{\boldsymbol{t}}\right\}_{t \in \mathbb{Z}}$ always denotes a standard normal distributed White Noise sequence.

We begin with a study of the power of the bootstrap test, where 1000 and 500 simulation runs are used for estimating the rejection probabilities under the null hypothesis and alternative, respectively, and 300 bootstrap replications are performed for the test (3.11). In order to investigate the approximation of the nominal level we consider the bivariate $\mathrm{MA}(1)$ and AR(1) models

$$
\begin{aligned}
& \boldsymbol{X}_{\boldsymbol{t}}=\boldsymbol{Z}_{\boldsymbol{t}}+\left(\begin{array}{cc}
\theta & 0.2 \\
0.2 & \theta
\end{array}\right) \boldsymbol{Z}_{\boldsymbol{t}-\mathbf{1}} \\
& \boldsymbol{X}_{\boldsymbol{t}}=\left(\begin{array}{cc}
\phi & 0.2 \\
0.2 & \phi
\end{array}\right) \boldsymbol{X}_{\boldsymbol{t}-\mathbf{1}}+\boldsymbol{Z}_{\boldsymbol{t}} .
\end{aligned}
$$

The simulated rejection frequencies for different values of the parameters $\theta$ and $\phi$ are given in Table 1 and we observe that the nominal level is underestimated for small sample sizes, but the approximation becomes better with increasing sample size. Next we study the power

\begin{tabular}{|c|c|c|c|c|c|c|c|c|}
\hline & \multicolumn{4}{|c|}{$\mathrm{H}_{0}$ : Model 6.1} & \multicolumn{3}{c|}{$\mathrm{H}_{0}$ : Model 6.2} \\
\hline & \multicolumn{2}{|c|}{$\theta=0.5$} & \multicolumn{2}{c|}{$\theta=-0.5$} & \multicolumn{2}{c|}{$\phi=0.5$} & \multicolumn{2}{c|}{$\phi=-0.5$} \\
\hline$T$ & $5 \%$ & $10 \%$ & $5 \%$ & $10 \%$ & $5 \%$ & $10 \%$ & $5 \%$ & $10 \%$ \\
\hline 128 & 0.018 & 0.049 & 0.019 & 0.047 & 0.021 & 0.061 & 0.020 & 0.059 \\
\hline 256 & 0.025 & 0.054 & 0.029 & 0.063 & 0.031 & 0.070 & 0.031 & 0.068 \\
\hline 512 & 0.043 & 0.081 & 0.039 & 0.088 & 0.034 & 0.075 & 0.040 & 0.083 \\
\hline
\end{tabular}

Table 1: Simulated nominal level of the test (3.11) for the models (6.1) and (6.2) with different choices of $\theta, \phi$ and $T$.

of the test (3.11) and compare it with the CUSUM type procedure proposed by Aue et al. (2009). This procedure is specifically designed to test for constancy of the covariance matrix $\operatorname{Cov}\left(\boldsymbol{X}_{t}, \boldsymbol{X}_{t}\right)$. We consider three bivariate models 


$$
\begin{aligned}
\boldsymbol{X}_{t, T} & =\sum_{l=0}^{K} 1_{\left[\left[b_{l} T\right]+1,\left\lfloor b_{l+1} T\right]\right]}(t)\left(\begin{array}{cc}
\phi_{l} & 0.1 \\
0.1 & \phi_{l}
\end{array}\right) \boldsymbol{X}_{t-1, T}+\boldsymbol{Z}_{t} \\
\boldsymbol{X}_{t, T} & =\sum_{l=0}^{K} 1_{\left[\left[b_{l} T\right]+1,\left\lfloor b_{l+1} T\right]\right]}(t)\left(\begin{array}{cc}
\theta_{l} & 0.1 \\
0.1 & \theta_{l}
\end{array}\right) \boldsymbol{Z}_{t-1}+\boldsymbol{Z}_{t} \\
\boldsymbol{X}_{t, T} & =\sum_{l=0}^{K} 1_{\left[\left[b_{l} T\right]+1,\left\lfloor b_{l+1} T\right]\right]}(t)\left(\begin{array}{cc}
\sigma_{l} & 0.2 \\
0.2 & \sigma_{l}
\end{array}\right) \boldsymbol{Z}_{t}
\end{aligned}
$$

for different choices of the number $K$ and location $\mathfrak{b}=\left(b_{1}, \ldots, b_{K}\right)$ of the break points and parameters $\Sigma:=\left(\sigma_{0}, \ldots, \sigma_{K}\right), \Phi:=\left(\phi_{0}, \ldots, \phi_{K}\right)$ and $\Theta:=\left(\theta_{0}, \ldots, \theta_{K}\right)$. The results are summarized in Table 2 ,

\begin{tabular}{|c|c|c|c|c|c|c|c|c|}
\hline & \multicolumn{2}{|c|}{} & \multicolumn{2}{c|}{$T=128$} & \multicolumn{2}{c|}{$T=256$} & \multicolumn{2}{c|}{$T=512$} \\
\hline & $\mathfrak{b}$ & parameter & $(3.11)$ & Aue & $(3.11)$ & Aue & $(3.11)$ & Aue \\
\hline \hline \multirow{2}{*}{6.3} & $\left(\frac{1}{4}, \frac{2}{3}, \frac{3}{4}\right)$ & $\Phi=(0.5,-0.5,0.5,-0.5)$ & 0.072 & 0.046 & 0.306 & 0.059 & 0.812 & 0.090 \\
& $\left(\frac{1}{2}\right)$ & $\Phi=(0.5,-0.5)$ & 0.078 & 0.047 & 0.234 & 0.146 & 0.702 & 0.337 \\
\hline \hline \multirow{2}{*}{6.4} & $\left(\frac{1}{4}, \frac{2}{3}, \frac{3}{4}\right)$ & $\Theta=(1,-1.5,1,-1.5)$ & 0.284 & 0.066 & 0.544 & 0.119 & 0.926 & 0.282 \\
& $\left(\frac{1}{2}\right)$ & $\Theta=(1,-1.5)$ & 0.258 & 0.257 & 0.542 & 0.620 & 0.850 & 0.965 \\
\hline \hline \multirow{2}{*}{6.4} & $\left(\frac{1}{4}, \frac{2}{3}, \frac{3}{4}\right)$ & $\Sigma=(1,2,1,0.5)$ & 1.000 & 0.213 & 1.000 & 0.989 & 1.000 & 1.000 \\
& $\left(\frac{1}{2}\right)$ & $\Sigma=(1,2)$ & 0.864 & 0.818 & 0.990 & 1.000 & 1.000 & 1.000 \\
& $\emptyset$ & $\Sigma=(1)$ & 0.026 & 0.033 & 0.029 & 0.048 & 0.041 & 0.047 \\
\hline
\end{tabular}

Table 2: Empirical rejection frequencies of the test (3.11) and the CUSUM type procedure of Aue et al. (2009). The nominal level is $\alpha=0.05$ and the models are defined in (6.3), (6.4) and (6.5), where different choices of break points $\mathfrak{b}=\left(b_{1}, \ldots, b_{K}\right)$ and AR-parameters $\Phi=\left(\phi_{0}, \ldots, \phi_{K}\right)$, MA-parameters $\Theta=\left(\theta_{0}, \ldots, \theta_{K}\right)$ and standard deviations $\Sigma=\left(\sigma_{0}, \ldots, \sigma_{K}\right)$ are considered.

We observe from the upper part of Table 2 that in the AR-model (6.3) the test proposed in this paper significantly outperforms the CUSUM method of Aue et al. (2009) in all cases under consideration. In the MA-model (6.4) the new test yields a substantially larger power if there exist multiple break points while the power is slightly smaller if there exists only one break point.

In the lower part of Table 2 we summarize the simulated power of the test (3.11) and the method proposed by Aue et al. (2009) for various configurations of the model (6.5). It can be observed that the new method significantly outperforms the method of Aue et al. (2009) 
for models with more than one break for small sample sizes. For the configurations featuring one break point we observe that the new method performs similarly for large sample sizes, while providing slightly better performance for small sample sizes. These observations are remarkable since model 6.5 defines a process with switching variance and the test of Aue et al. (2009) is particularly designed for detecting this structure. Since our approach is able to detect a much wider class of alternatives one might expect a loss in power compared to a procedure which is specifically constructed to test for such changes. Surprisingly, this does not seem to be the case.

Next we illustrate the performance of the procedure for the localization of the break points. For this purpose we simulated data from model $(4.2)$ and applied the new method to detect and estimate the location of the change points. We repeated this 100 times and for each trial we obtained an estimate $\hat{\mathfrak{b}}=\left(\hat{b}_{1}, \ldots, \hat{b}_{\hat{K}}\right)$ of the location $\mathfrak{b}=\left(b_{1}, \ldots, b_{K}\right)$. The histograms in Figure 3 present the obtained empirical distribution of the estimated break points for different sample sizes $T$. We observe that all histograms are centered at the true break points and that the variation decreases with increasing sample size.

We conclude this section with an illustration of the procedures in a multivariate data example. For this purpose we investigate five stock ETFs (Exchange Traded Funds), namely Materials Select Sector SPDR (XLB), Consumer Staples Select Sector SPDR (XLP), Utilities Select Sector SPDR (XLU), Financial Select Sector SPDR (XLF) and Energy Select Sector SPDR (XLE). In Figure 5 the five dimensional time series $\left\{\boldsymbol{X}_{\boldsymbol{t}}\right\}_{t=1, \ldots, 700}$ of the daily log returns corresponding to the five ETFs between February 2010 and November 2012 is displayed. The new method yields the following results: The test (3.11) for structural breaks is highly significant and rejects the null hypothesis with a $p$-value of zero. In a second step we use the detection procedure with $N=64$ and detect four break points at dates 29/04/2010, 03/08/2010, 03/08/2011, and 01/12/2011. We then also applied the refined analysis in order to identify the components where the change point is present. The results are displayed in Figure 4 and 5. Note that at the third break point 03/08/2011 each component 


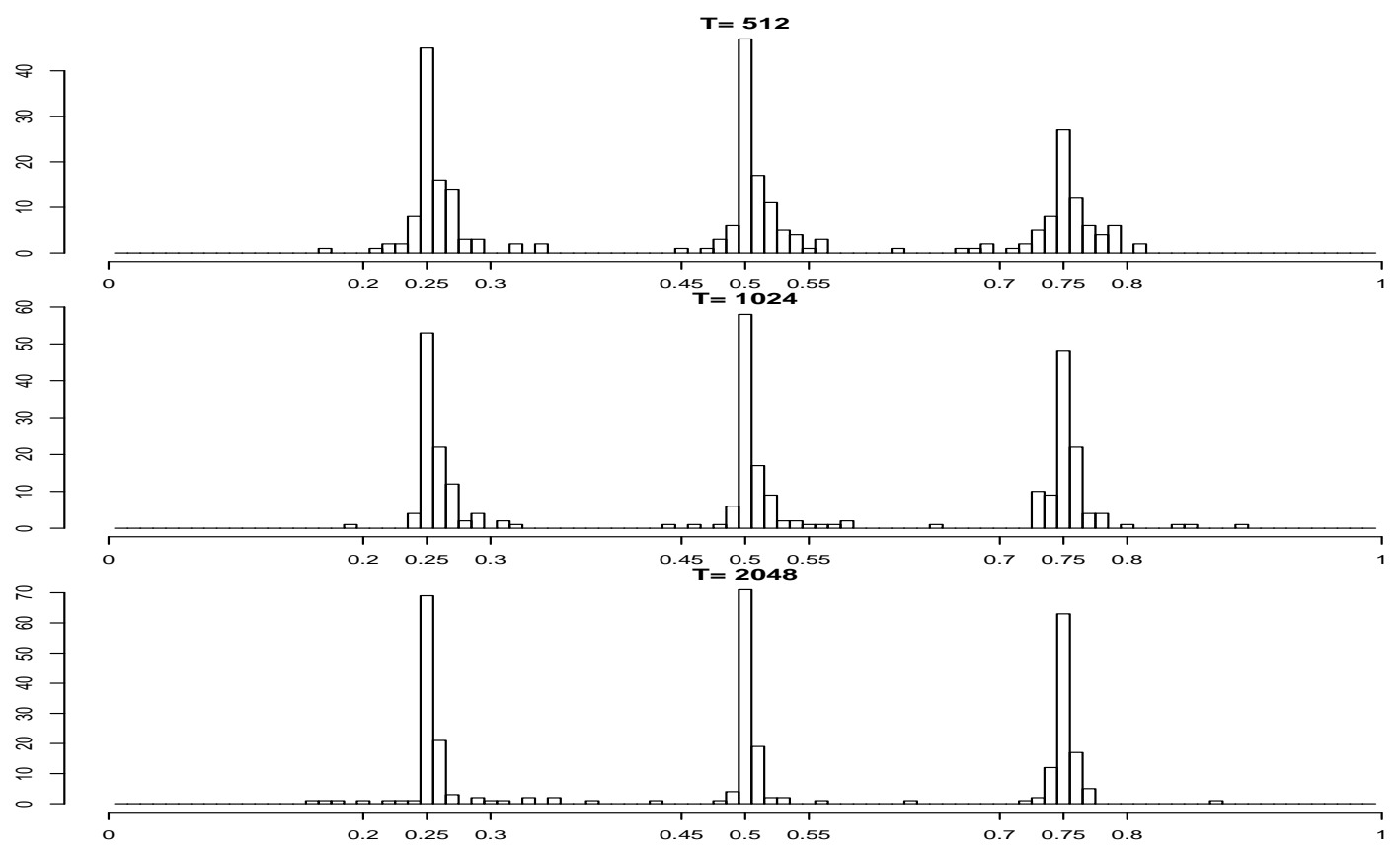

Figure 3: Histograms for the empirical distribution of $\hat{\mathfrak{b}}=\left(\hat{b}_{1}, \ldots, \hat{b}_{\hat{K}}\right)$ based on 100 simulation runs of model 4.2 for sample sizes $T \in\{512,1024,2048\}$.

$N^{\gamma} \sup _{\omega \in[0,1]}\left|\left[\hat{\mathbf{D}}_{T}\left(\hat{b}_{i}, \omega\right)\right]_{a, b}\right|$ surpasses the respective critical threshold $\boldsymbol{\varepsilon}_{a, b, T}\left(\hat{b}_{i}\right)$ and we conclude that each component of the spectral density exhibits a change point. On the other hand, for the other three break points only some entries of this matrix exceed the threshold sequence and we therefore detect a structural break only in some components of the spectral density matrix.

Acknowledgements This work has been supported in part by the Collaborative Research Center "Statistical modeling of nonlinear dynamic processes" (SFB 823, Teilprojekt C1) of the German Research Foundation (DFG).

\section{References}

Adak, S. (1998). Time-dependent spectral analysis of nonstationary time series. Journal of the American Statistical Association, 93(444):1488-1501.

Aue, A., Hörmann, S., Horvath, L., and Reimherr, M. (2009). Break detection in the covari- 


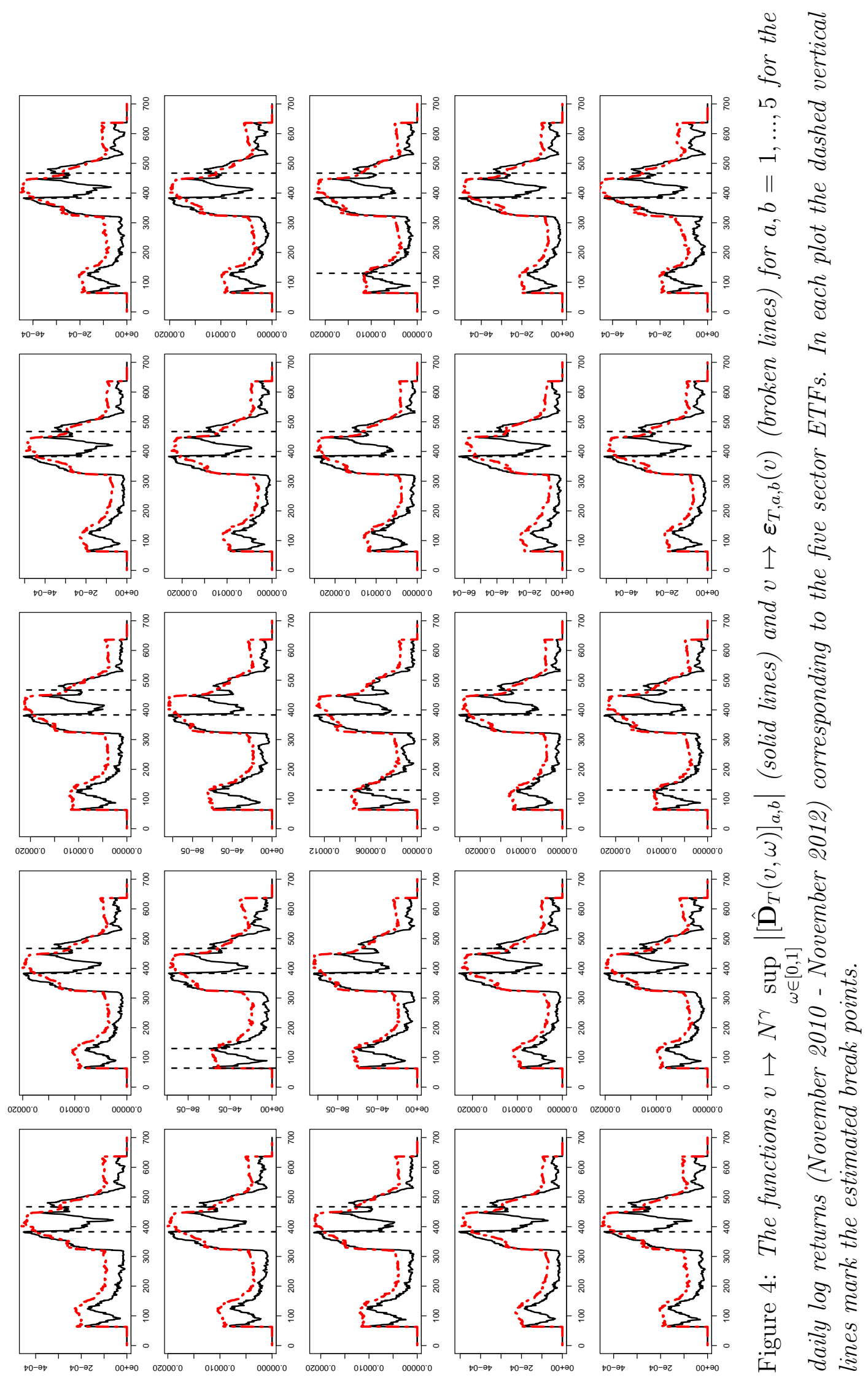



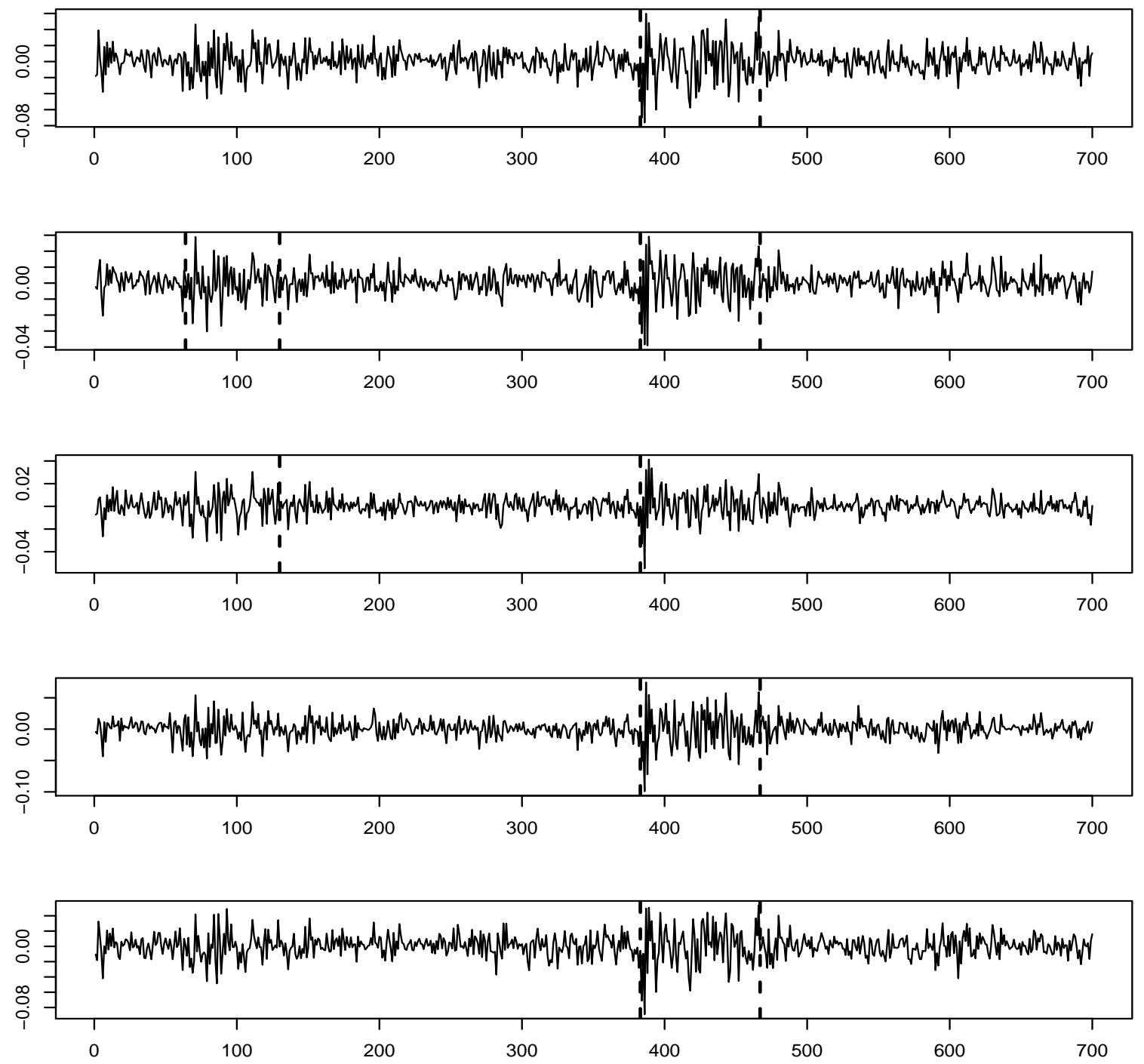

Figure 5: The log returns of the sector ETFs. In each plot the vertical lines mark the estimated break points of the univariate process. 
ance structure of multivariate time series models. Annals of Statistics, 37(6):4046-4087.

Bai, J. (1994). Least squares estimation of a shift in linear processes. Journal of Time Series Analysis, 15(5):453-472.

Banerjee, A., Lumsdaine, R., and Stock, J. (1992). Recursive and sequential tests of the unit-root and trend-break hypotheses: Theory and international evidence. Journal of Business 83 Economic Statistics, 10(3):271-287.

Berg, A., Paparoditis, E., and Politis, D. N. (2010). A bootstrap test for time series linearity. Journal of Statistical Planning and Inference, 140:3841-3857.

Brillinger, D. R. (1981). Time Series: Data Analysis and Theory. McGraw Hill, New York.

Chen, J. and Gupta, A. K. (1997). Testing and locating variance changepoints with application to stock prices. Journal of the American Statistical Association, 92(438):739-747.

Chen, Y., Härdle, W., and Pigorsch, U. (2010). Localized realized volatility modeling. Journal of the American Statistical Association, 105(492):1376-1393.

Choi, E. and Hall, P. (2000). Bootstrap confidence regions computed from autoregressions of arbitrary order. Journal of the Royal Statistical Society, Series B, 62:461-477.

Dahlhaus, R. (1988). Empirical spectral processes and their applications to time series analysis. Stochastic Process and their Applications, 30:69-83.

Dahlhaus, R. (1997). Fitting time series models to nonstationary processes. Annals of Statistics, 25(1):1-37.

Davis, R. A., Lee, T. C. M., and Rodriguez-Yam, G. A. (2006). Structural break estimation for nonstationary time series models. Journal of the American Statistical Association, 101(473):223-239. 
Eichler, M. (2008). Testing nonparametric and semiparametric hypotheses in vector stationary processes. Journal of Multivariate Analysis, 99:968-1009.

Fan, J. (1994). Test of significance based on wavelet thresholding and neyman's truncation. Journal of the American Statistical Association, 91(434):674-688.

Fryzlewicz, P. (2007). Unbalanced haar technique for nonparametric function estimation. Journal of the American Statistical Association, 102(480):1318-1327.

Fryzlewicz, P. (2012). Wild binary segmentation for multiple change-point detection. http: //stats.lse.ac.uk/fryzlewicz/wbs/wbs.pdf.

Fryzlewicz, P., Sapatinas, T., and Subba Rao, S. (2006). A Haar-Fisz technique for locally stationary volatility estimation. Biometrika, 93:687-704.

Giraitis, L. and Leipus, R. (1990). Functional clt for nonparametric estimates of the spectrum and change-point problem for a spectral function. Lithuanian Mathematical Journal, $30(4): 302-322$.

Goncalves, S. and Kilian, L. (2007). Asymptotic and bootstrap inference for $\operatorname{ar}(\infty)$ processes with conditional heteroskedasticity. Econometric Reviews, 26:609-641.

Hannan, E. and Kavalieris, L. (1986). Regression, autoregression models. Journal of Time Series Analysis, 7(1):27-49.

Inclan, C. and Tiao, G. C. (1994). Use of cumulative sums of squares for retrospective detection of changes of variance. Journal of the American Statistical Association, 89(427):913923.

James, B., James, K. L., and Siegmund, D. (1987). Tests for a change-point. Biometrika, $74(1): 71-83$.

Jansen, B., Hasman, A., and Lenten, R. (1981). Piecewise eeg analysis: An objective evaluation. International Journal of Bio-Medical Computing, 12:12-27. 
Jolley, L. (1961). Summation of Series.

Kreiss, J.-P. (1988). Asymptotic statistical inference for a class of stochastic processes. Habilitationsschrift, Fachbereich Mathematik, Universität Hamburg.

Kreiss, J. P. and Paparoditis, E. (2012). The hybrid wild bootstrap for time series. to appear in: Journal of the American Statistical Association.

Kreiss, J.-P., Paparoditis, E., and Politis, D. N. (2011). On the range of the validity of the autoregressive sieve bootstrap. To appear in: Annals of statistics.

Lavielle, M. and Ludena, C. (2000). The multiple change-points problem for the spectral distribution. Bernoulli, 6(5):845-869.

Lee, S., Ha, J., Na, O., and Na, S. (2003). The cusum test for parameter change in time series models. Scandinavian Journal of Statistics, 30:781-796.

Lee, S. and Park, S. (2001). The cusum of squares test for scale changes in infinite order moving average processes. Scandinavian Journal of Statistics, 28(4):625-644.

Newey, W. K. (1991). Uniform convergence in probability and stochastic equicontinuity. Econometrica, 59(4):1161-1167.

Ombao, H. C., Raz, J. A., von Sachs, R., and Malow, B. A. (2001). Automatic statistical analysis of bivariate nonstationary time series. Journal of the American Statistical Association, 96(454):543-560.

Paparoditis, E. (2010). Validating stationarity assumptions in time series analysis by rolling local periodograms. Journal of the American Statistical Association, 105(490):839-851.

Preuß, P. and Vetter, M. (2012). Discriminating between long-range dependence and nonstationarity. http://www.ruhr-uni-bochum.de/imperia/md/content/mathematik3/ publications/longmemory24092012.pdf. 
Preuß, P., Vetter, M., and Dette, H. (2012). A test for stationarity based on empirical processes. to appear in Bernoulli.

Sen, A. and Srivastava, M. S. (1975). On tests for detecting change in mean. Annals of Statistics, 3(1):98-108.

Starica, C. and Granger, C. (2005). Nonstationarities in stock returns. The Review of Economics and Statistics, 87:503-522.

van der Vaart, A. and Wellner, J. (1996). Weak Convergence and Empirical Processes. Springer, Berlin.

Vostrikova, L. J. (1981). Detecting 'disorder' in multidimensional random processes. Soviet Mathematics Doklady, 24:55-59.

Whittle, P. (1951). Hypothesis Testing in Time Series Analysis. Uppsala: Almqvist and Wiksell.

\section{Appendix I: Sketch of the proofs}

For the sake of brevity and in order to improve the readability we restrict ourselves to the main ideas of the proofs in this section and present all technical details in an additional Appendix thereafter.

Proof of Theorem 3.1 For notational convenience we restrict ourselves to the case $d=1$, since the more general case is treated completely analogously using linearity arguments and the independence of the components of $\boldsymbol{Z}_{t}$. Throughout this chapter $C$ denotes a universal constant, which does not depend on the sample size and can vary from line to line in the calculations. 
Proof of part a): For the proof of (3.5) it is sufficient to show the following two claims: [see Theorem 1.5.4 and 1.5.7 in van der Vaart and Wellner (1996)]:

(1) For every $k \in \mathbb{N}$ and $y_{1}:=\left(v_{1}, \omega_{1}\right), \ldots, y_{k}:=\left(v_{k}, \omega_{k}\right) \in[0,1]$ we have

$$
\sqrt{N}\left[\hat{D}_{T}\left(y_{1}\right), \ldots, \hat{D}_{T}\left(y_{k}\right)\right] \Rightarrow\left[G\left(y_{1}\right), \ldots, G\left(y_{k}\right)\right]
$$

(2) For every $\eta, \varepsilon>0$ there exists a $\delta>0$ such that

$$
\lim _{T \rightarrow \infty} P\left(\sup _{\left(y_{1}, y_{2}\right) \in[0,1]^{2}: d_{2}\left(y_{1}, y_{2}\right)<\delta} N^{1 / 2}\left|\hat{D}_{T}\left(y_{1}\right)-\hat{D}_{T}\left(y_{2}\right)\right|>\eta\right)<\varepsilon
$$

where $d_{2}\left(y_{1}, y_{2}\right)$ denotes the euclidean distance between $y_{1}=\left(v_{1}, \omega_{1}\right)$ and $y_{2}=\left(v_{2}, \omega_{2}\right)$.

Proof of (7.1): The assertion follows if we are able to show that, for each $k \in \mathbb{N}$ and each $y_{1}=\left(v_{1}, \omega_{1}\right), \ldots, y_{k}=\left(v_{k}, \omega_{k}\right)$, all cumulants of the random vector $\left[\sqrt{N} \hat{D}_{T}\left(y_{i}\right)\right]_{i=1, \ldots, k}$ converge to the corresponding cumulants of the vector $\left[G\left(y_{i}\right)\right]_{i=1, \ldots, k}$. We thus have to show for all $y=(v, \omega), y_{1}, \ldots, y_{l} \in[0,1]^{2}$ :

(i) $\mathbb{E}\left(\sqrt{N} \hat{D}_{T}(y)\right)=o(1)$.

(ii) $\operatorname{Cov}\left(\sqrt{N} \hat{D}_{T}\left(y_{1}\right), \sqrt{N} \hat{D}_{T}\left(y_{2}\right)\right)=\operatorname{Cov}\left(G\left(y_{1}\right), G\left(y_{2}\right)\right)+o(1)$.

(iii) $\operatorname{cum}\left(\sqrt{N} \hat{D}_{T}\left(y_{1}\right), \ldots, \sqrt{N} \hat{D}_{T}\left(y_{l}\right)\right)=o(1)$.

For this purpose we define for $y=(v, \omega)$

$$
\phi_{y, T}(j, \lambda):=1_{\left[0, \frac{2 \pi\lfloor\omega N / 2\rfloor}{N}\right]}(\lambda)\left[1_{\{\lfloor u(v, T) T\rfloor+N / 2\}}(j)-1_{\{\lfloor u(v, T) T\rfloor-N / 2\}}(j)\right],
$$

where the function $u$ is defined by $u(v, T)=v$ if $N / T \leq v \leq 1-N / T, N / T$ if $v<N / T$, and $1-N / T$ if $v>1-N / T$. This notation implies the representation

$$
\hat{D}_{T}(y)=\frac{1}{N} \sum_{j=1}^{T} \sum_{k=1}^{N / 2} \phi_{y, T}\left(j, \lambda_{k}\right) I_{N}\left(\frac{j}{T}, \lambda_{k}\right) .
$$


Additionally, we set $D_{N, T}(y):=\frac{T}{N}\left(\int_{0}^{\omega \pi} \int_{v}^{v+N / T} f(u, \lambda) d u d \lambda-\int_{0}^{\omega \pi} \int_{v-N / T}^{v} f(u, \lambda) d u d \lambda\right)$, and start with a proof of

$$
\mathbb{E}\left(\sqrt{N}\left(\hat{D}_{T}(y)-D_{N, T}(y)\right)\right)=o(1)
$$

from which (i) follows directly because of $D_{N, T}(y) \equiv 0$ under the null hypothesis [we treat the more general case since (7.5) is also required in the proof of part b)]. By writing $\psi_{l}(t / T):=\Psi_{l}(t / T)$ we get $(y=(v, \omega))$

$$
\begin{aligned}
& \mathbb{E}\left(\hat{D}_{T}(y)\right)=\frac{1}{2 \pi N^{2}} \sum_{j=1}^{T} \sum_{k=1}^{N / 2} \phi_{y, T}\left(j, \lambda_{k}\right) \sum_{p, q=0}^{N-1} e^{-i \lambda_{k}(p-q)} \mathbb{E}\left(X_{j-\frac{N}{2}+1+p} X_{j-\frac{N}{2}+1+q}\right) \\
= & \frac{1}{2 \pi N^{2}} \sum_{j=1}^{T} \sum_{k=1}^{N / 2} \phi_{y, T}\left(j, \lambda_{k}\right) \sum_{l, m=0}^{\infty} \psi_{l}\left(\frac{j-\frac{N}{2}+1+p}{T}\right) \psi_{m}\left(\frac{j-\frac{N}{2}+1+q}{T}\right) \sum_{p, q=0}^{N-1} e^{-i \lambda_{k}(p-q)} \\
& \times \mathbb{E}\left(Z_{j-\frac{N}{2}+1+p-l} Z_{j-\frac{N}{2}+1+q-m}\right)
\end{aligned}
$$

and by using the identity $\mathbb{E}\left(Z_{i} Z_{j}\right)=\delta_{i j}$ [here and throughout this paper $\delta_{i j}$ denotes the Kronecker symbol] we obtain that the restriction $q=p-l+m$ has to hold such that the respective summands in $(7.6)$ do not vanish. If we furthermore define $A_{T, 1}(v):=\{\lfloor v T\rfloor-$ $N / 2,\lfloor v T\rfloor+N / 2\}$, we obtain for 7.6

$$
\begin{aligned}
& \frac{1}{2 \pi N^{2}} \sum_{j \in A_{T, 1}(v)} \sum_{k=1}^{N / 2} \phi_{y, T}\left(j, \lambda_{k}\right) \sum_{l, m=0}^{\infty} \sum_{\substack{p=0 \\
0 \leq p-l+m \leq N-1}}^{N-1} \\
& \times \psi_{l}\left(\frac{j-\frac{N}{2}+1+p}{T}\right) \psi_{m}\left(\frac{j-\frac{N}{2}+1+p-l+m}{T}\right) e^{-i \lambda_{k}(m-l)} \\
= & \frac{1}{2 \pi N^{2}} \sum_{j \in A_{T, 1}(v)} \sum_{k=1}^{N / 2} \phi_{y, T}\left(j, \lambda_{k}\right) \sum_{l, m=0}^{\infty} \sum_{\substack{p=0 \\
0 \leq p-l+m \leq N-1}}^{N-1}\left\{\psi_{l}\left(\frac{j-\frac{N}{2}+1+p}{T}\right) \psi_{m}\left(\frac{j-\frac{N}{2}+1+p}{T}\right)\right. \\
+ & \left.\psi_{l}\left(\frac{j-\frac{N}{2}+1+p}{T}\right)\left(\psi_{m}\left(\frac{j-\frac{N}{2}+1+p-l+m}{T}\right)-\psi_{m}\left(\frac{j-\frac{N}{2}+1+p}{T}\right)\right)\right\} e^{-i \lambda_{k}(m-l)} \\
= & : E_{1, T}(y)+E_{2, T}(y),
\end{aligned}
$$

where $E_{1, T}$ and $E_{2, T}$ are defined in an obvious manner. The assertion now follows from 
(ia) $E_{1, T}(y)=D_{N, T}(y)+O(1 / N)$.

(ib) $E_{2, T}(y)=o(1 / \sqrt{N})$

which are established in Appendix II. The proof of (ii) and (iii) are also given in Appendix II.

Proof of 7.2 : Observing

$$
\begin{aligned}
& \sqrt{N} \hat{D}_{T}(y)=\frac{1}{\sqrt{N}} \sum_{k=1}^{\lfloor\omega N / 2\rfloor} I_{N}\left(u(v, T)+N /(2 T), \lambda_{k}\right)-\frac{1}{\sqrt{N}} \sum_{k=1}^{\lfloor\omega N / 2\rfloor} I_{N}\left(u(v, T)-N /(2 T), \lambda_{k}\right) \\
& =: \sqrt{N} \hat{D}_{T}^{(1)}(y)-\sqrt{N} \hat{D}_{T}^{(2)}(y),
\end{aligned}
$$

it is sufficient to show asymptotic stochastic equicontinuity for the processes $\left\{\sqrt{N} \hat{D}_{T}^{(1)}(y)\right\}_{y \in[0,1]^{2}}$ and $\left\{\sqrt{N} \hat{D}_{T}^{(2)}(y)\right\}_{y \in[0,1]^{2}}$. We only present the proof for the first summand in (7.8) and note that stochastic equicontinuity for the second term can be shown analogously. With the notation $\phi_{y, T}^{(1)}(j, \lambda):=1_{\left[0, \frac{2 \pi\lfloor\omega N / 2\rfloor}{N}\right]}(\lambda) 1_{\{\lfloor u(v, T) \times T\rfloor+N / 2\}}(j)$, we obtain the representation $\hat{D}_{T}^{(1)}(y)=\frac{1}{N} \sum_{j=1}^{T} \sum_{k=1}^{N / 2} \phi_{y, T}^{(1)}\left(j, \lambda_{k}\right) I_{N}\left(j / T, \lambda_{k}\right)$. For $y_{i}=\left(v_{i}, \omega_{i}\right)(i=1,2)$ we define a semi-metric $d_{T}\left(y_{1}, y_{2}\right):=\sqrt{\left|\omega_{2}-\omega_{1}\right|+\left\lfloor\left\lfloor v_{1} T\right\rfloor-\left\lfloor v_{2} T\right\rfloor \mid / N\right.}$ on the set $\mathcal{P}_{T}:=$ $\{0,1 / T, 2 / T, \ldots, 1-N / T\} \times\{1 / N, \ldots, 1-1 / N, 1\}$. This yields

$$
\Delta_{\delta, \eta}:=\mathbb{P}\left(\sup _{\substack{y_{i} \in[0,1]^{2} \\ d_{2}\left(y_{1}, y_{2}\right)<\delta}} \sqrt{N}\left|\hat{D}_{T}^{(1)}\left(y_{1}\right)-\hat{D}_{T}^{(1)}\left(y_{2}\right)\right|>\eta\right)=\mathbb{P}\left(\sup _{\substack{y_{i} \in \mathcal{P}_{T} \\ d_{2}\left(y_{1}, y_{2}\right)<\delta}} \sqrt{N}\left|\hat{D}_{T}^{(1)}\left(y_{1}\right)-\hat{D}_{T}^{(1)}\left(y_{2}\right)\right|>\eta\right)
$$

and it is easy to verify that, for a fixed $\delta>0$, there exists a $\delta^{\prime}>0$ [with $\delta^{\prime}(\delta) \rightarrow 0$ as $\left.\delta \rightarrow 0\right]$ such that

$$
\Delta_{\delta, \eta} \leq \mathbb{P}\left(\sup _{y_{i} \in \mathcal{P}_{T}: d_{T}\left(y_{1}, y_{2}\right)<\delta^{\prime}} \sqrt{N}\left|\hat{D}_{T}^{(1)}\left(y_{1}\right)-\hat{D}_{T}^{(1)}\left(y_{2}\right)\right|>\eta\right)
$$

is fulfilled if $T$ is sufficiently large. So it suffices to prove that the probability on the right hand side of $(7.9)$ can be made arbitrarily small if $T$ is sufficiently large. For this purpose let $C\left(u, d_{T}, \mathcal{P}_{T}\right)$ denote the covering number of $\mathcal{P}_{T}$ with respect to the semi-metric $d_{T}(\cdot, \cdot)$ and define the corresponding covering integral of $\mathcal{P}_{T}$ by $J_{T}(\kappa):=\int_{0}^{\kappa}\left[\log \left(\frac{48 C\left(u, d_{T}, \mathcal{P}_{T}\right)^{2}}{u}\right)\right]^{2} d u$. 
In Appendix II we establish the assertions

$$
\begin{aligned}
& \lim _{\kappa \rightarrow 0} \lim _{T \rightarrow \infty} J_{T}(\kappa)=0 \\
& \mathbb{E}\left(N^{k / 2}\left(\hat{D}_{T}^{(1)}\left(y_{1}\right)-\hat{D}_{T}^{(1)}\left(y_{2}\right)\right)^{k}\right) \leq(2 k) ! C^{k} d_{T}\left(y_{1}, y_{2}\right)^{k}
\end{aligned}
$$

for a constant $C \in \mathbb{R}^{+}$and all $y_{1}, y_{2} \in[0,1]^{2}$ and even integers $k \in \mathbb{N}$. Therefore it follows, by similar arguments as given in Dahlhaus (1988), that

$$
\mathbb{P}\left(\sup _{\substack{y_{i} \in \mathcal{P}_{T} \\ d_{T}\left(y_{1}, y_{2}\right)<\delta^{\prime}}} \sqrt{N}\left|\hat{D}_{T}^{(1)}\left(y_{1}\right)-\hat{D}_{T}^{(1)}\left(y_{2}\right)\right|>\eta\right)<\varepsilon
$$

for $T$ sufficiently large and sufficiently small $\delta^{\prime}=\delta^{\prime}(\delta)>0$, which proves stochastic equicontinuity.

Proof of part b): Under the alternative, there exist for all $r \in\{1, \ldots, K\}$ an $\omega_{r}$ such that $\left|D\left(b_{r}, \omega_{r}\right)\right|>0$. Note that, the proof of part a) does not rely on the property that the functions $\psi_{l}(u)$ are constant. In fact, only the treatment of the expectation [i.e. part (1) (i)] is slightly easier if $\psi_{l}(u)=\psi_{l}$. However, in this case we proved the more general claim (7.5). So, by following the proofs of $(i i)$ and (iii) in the proof of part a) and employing (7.5), we obtain $N^{1 / 2}\left\|\hat{D}_{T}(y)-D_{N, T}(y)\right\|_{\infty}=O_{P}(1)$, which directly yields the assertion.

\section{Proof of Theorem 3.2; See Appendix II.}

Proof of Theorem 3.5 As in the previous proof we restrict ourselves without loss of generality to the case $d=1$. Furthermore we suppress the $\operatorname{argument} T$, when referring to the sequence $p=p(T)$. Because of Assumption 3.3, we obtain the $M A(\infty)$ representation $X_{t, T}^{*}=\sum_{l=0}^{\infty} \hat{\psi}_{l}^{A R}(p) Z_{t-l}^{*}$ for $T$ and $p(T)$ sufficiently large [see Section 3 of Preuß et al. (2012) for more details]. Note that this representation corresponds to a process without structural breaks and that in the proof of Theorem 3.1a) all error terms can be bounded by

$$
\frac{\left(\sum_{m=0}^{\infty}\left|\psi_{m}\right|\right)^{q_{1}}\left(\sum_{l=0}^{\infty} l\left|\psi_{l}\right|\right)^{q_{2}}}{N}=O(1 / N)
$$


where $q_{1}, q_{2} \in \mathbb{N}$ and the equality is a consequence of (3.4). So the proof of Theorem 3.5 follows in the same way as the proof of Theorem 3.1 a) if we show that the (now random) errors terms are of order $O_{P}(1 / N)$. However, it was shown in Theorem 3.2 of Preuß et al. (2012) that $\left(\sum_{m=0}^{\infty}\left|\hat{\psi}_{m}^{A R}(p)\right|\right)^{q_{1}}\left(\sum_{l=0}^{\infty} l\left|\hat{\psi}_{l}^{A R}(p)\right|\right)^{q_{2}}=O_{P}(1)$ holds for all $q_{1}, q_{2} \in \mathbb{I}$, which directly yields the claim.

Proof of Theorem 3.6: see Appendix II.

Proof of Theorem 4.1 For a Proof of part a) note that $\sup _{\omega \in[0,1]}\left|\left[\boldsymbol{D}_{N, T}(v, \omega)\right]_{a, b}\right|=0$ for $v \in \overline{\mathcal{I}}_{T, a, b}\left(b_{1}, \ldots, b_{K}\right)$, and observe (4.7). This yields for all $a, b \in\{1, \ldots, d\}$ and sufficiently large $N, T$

$$
\begin{aligned}
& \mathbb{P}\left(\bigcup_{v \in \overline{\mathcal{I}}_{T, a, b}\left(b_{1}, \ldots, b_{K}\right)}\left\{N^{\gamma} \sup _{\omega \in[0,1]}\left|\left[\hat{\boldsymbol{D}}_{T}(v, \omega)\right]_{a, b}\right|>\boldsymbol{\varepsilon}_{T, a, b}(v)\right\}\right) \\
\leq & \mathbb{P}\left(N^{\gamma} \sup _{v \in \overline{\mathcal{I}}_{T, a, b}\left(b_{1}, \ldots, b_{K}\right)} \sup _{\omega \in[0,1]}\left|\left[\hat{\boldsymbol{D}}_{T}(v, \omega)\right]_{a, b}-\left[\boldsymbol{D}_{N, T}(v, \omega)\right]_{a, b}\right|>C / 2\right) \\
\leq & \mathbb{P}\left(N^{\gamma} \sup _{v \in[0,1]} \sup _{\omega \in[0,1]}\left\|\hat{\boldsymbol{D}}_{T}(v, \omega)-\boldsymbol{D}_{N, T}(v, \omega)\right\|_{\infty}>C / 2\right) \stackrel{T \rightarrow \infty}{\longrightarrow} 0 .
\end{aligned}
$$

where we used Theorem 3.1 (if assumption (2) is fulfilled) and 3.2 (if assumption (1) is fulfilled). Part b) is a direct consequence of Theorem 3.1b) and $3.2 \mathrm{~b}$ ), which imply for $r=1, \ldots, K$ and $(a, b) \in B\left(b_{r}\right): \mathbb{P}\left(\sup _{\omega \in[0,1]} N^{\gamma}\left|\left[\hat{\boldsymbol{D}}_{T}\left(b_{r}, \omega\right)\right]_{a, b}\right|>\boldsymbol{\varepsilon}_{T a, b}\left(b_{r}\right)\right) \rightarrow 1$. 


\section{Appendix II: technical details}

\subsection{Proof of (ia) and (ib) in the proof of part (a) Theorem 3.1}

For a proof of (ia), we note that by 3.4 we can drop the restriction in the summation with respect to $p$ by making an error of order $O(1 / N)$. Thus we obtain for $E_{1, T}$

$$
\begin{aligned}
& \frac{1}{2 \pi N^{2}} \sum_{j \in A_{T, 1}(v)} \sum_{k=1}^{N / 2} \phi_{y, T}\left(j, \lambda_{k}\right) \sum_{l, m=0}^{\infty} \sum_{p=1}^{N-1} \psi_{l}\left(\frac{j-\frac{N}{2}+1+p}{T}\right) \psi_{m}\left(\frac{j-\frac{N}{2}+1+p}{T}\right) e^{-i \lambda_{k}(m-l)} \\
& +O(1 / N) \\
= & \frac{1}{N} \sum_{j \in A_{T, 1}(v)} \sum_{k=1}^{N / 2} \phi_{y, T}\left(j, \lambda_{k}\right) \frac{1}{2 \pi} \sum_{l, m=0}^{\infty} \frac{T}{N} \int_{j / T-\frac{N}{2 T}+\frac{1}{T}}^{j / T+\frac{N}{2 T}} \psi_{l}(u) \psi_{m}(u) d u \quad e^{-i \lambda_{k}(m-l)} d u+O(1 / N) \\
= & D_{N, T}(y)+O(1 / N),
\end{aligned}
$$

where the second equality follows with the piecewise constancy of the functions $\psi_{l}(u)$. For (ib) we get

$$
\left|E_{2, T}(y)\right| \leq C \sum_{j \in A_{T, 1}(v)} \sum_{\substack{l, m=0 \\|l-m| \leq N}}^{\infty}\left|\int_{j / T-\frac{N}{2 T}+\frac{1}{T}}^{j / T+\frac{N}{2 T}} \psi_{l}(u)\left(\psi_{m}\left(u+\frac{m-l}{T}\right)-\psi_{m}(u)\right) d u\right|+O(1 / N)
$$

where the summation can be restricted to indices satisfying $|l-m| \leq N$, which follows directly from the restriction on $p$. By employing that

$$
\int_{a}^{b} f(x) g(x+y) d x-\int_{a}^{b} f(x) g(x) d x \leq C|y| \sup _{z \in[a-|y|, b+|y|]}|f(z)| \sup _{z_{1}, z_{2} \in[a-|y|, b+|y|]}\left|g\left(z_{1}\right)-g\left(z_{2}\right)\right|
$$

holds for all piecewise constant functions $f, g$ exhibiting only finitely many points of discontinuity on the interval $[a-|y|, b+|y|]$, we obtain

$$
\left|E_{2, T}(y) \leq\right| C \sum_{j \in A_{T, 1}(v)} \sum_{\substack{l, m=0 \\|l-m| \leq N}}^{\infty} \frac{|m-l|}{T} \sup _{u \in[0,1]}\left|\psi_{l}(u)\right| \sup _{u \in[0,1]}\left|\psi_{m}(u)\right|+O(1 / N)=o(1 / \sqrt{N}) .
$$




\subsection{Proof of (ii) and (iii) in the proof of part (a) of Theorem 3.1}

For the proof of part (ii) note that, under the null hypothesis of no structural breaks, the quantities $\psi_{l}(t / T)$ do not depend on the rescaled time $t / T$ and we can work with the representation

$$
X_{t, T}=\sum_{l=0}^{\infty} \psi_{l} Z_{t-l}
$$

Without loss of generality we assume that $v_{1} \leq v_{2}$ holds and define the set

$$
A_{T, 2}\left(v_{1}, v_{2}\right):=\left\{\left\lfloor v_{1} T\right\rfloor-N / 2,\left\lfloor v_{1} T\right\rfloor+N / 2\right\} \times\left\{\left\lfloor v_{2} T\right\rfloor-N / 2,\left\lfloor v_{2} T\right\rfloor+N / 2\right\} .
$$

We then obtain for $\omega_{1}, \omega_{2} \in[0,1]$

$$
\begin{aligned}
& \operatorname{Cov}\left(\sqrt{N} \hat{D}_{T}\left(y_{1}\right), \sqrt{N} \hat{D}_{T}\left(y_{2}\right)\right) \\
= & \frac{1}{N} \sum_{\left(j_{1}, j_{2}\right) \in A_{T, 2}\left(v_{1}, v_{2}\right)} \sum_{k_{1}, k_{2}=1}^{N / 2} \phi_{y_{1}, T}\left(j_{1}, \lambda_{k_{1}}\right) \phi_{y_{2}, T}\left(j_{2}, \lambda_{k_{2}}\right) \operatorname{cum}\left(I_{N}\left(\frac{j_{1}}{T}, \lambda_{k_{1}}\right), I_{N}\left(\frac{j_{2}}{T}, \lambda_{k_{2}}\right)\right) \\
= & \frac{1}{(2 \pi)^{2} N^{3}} \sum_{\left(j_{1}, j_{2}\right) \in A_{T, 2}\left(v_{1}, v_{2}\right)} \sum_{k_{1}, k_{2}=1}^{N / 2} \phi_{y_{1}, T}\left(j_{1}, \lambda_{k_{1}}\right) \phi_{y_{2}, T}\left(j_{2}, \lambda_{k_{2}}\right) \sum_{p_{1}, p_{2}=0}^{N-1} \sum_{q_{1}, q_{2}=0}^{N-1} \sum_{l, m, n, o=0}^{\infty} \psi_{l} \psi_{m} \\
& \times \psi_{n} \psi_{o} e^{-i \lambda_{k_{1}}\left(p_{1}-q_{1}\right)} e^{-i \lambda_{k_{2}}\left(p_{2}-q_{2}\right)} \operatorname{cum}\left(Z_{j_{1}+p_{1}+1-l} Z_{j_{1}+q_{1}+1-m}, Z_{j_{2}+p_{2}+1-n} Z_{j_{2}+q_{2}+1-o}\right) \\
= & \sum_{\left(j_{1}, j_{2}\right) \in A_{T, 2}\left(v_{1}, v_{2}\right)} B_{T}\left(j_{1}, j_{2}\right),
\end{aligned}
$$

where $B_{T}\left(j_{1}, j_{2}\right)$ is defined in an obvious manner. Using

$$
\operatorname{cum}\left(Z_{a} Z_{b}, Z_{c} Z_{d}\right)=\operatorname{cum}\left(Z_{a}, Z_{d}\right) \operatorname{cum}\left(Z_{b}, Z_{c}\right)+\operatorname{cum}\left(Z_{a}, Z_{c}\right) \operatorname{cum}\left(Z_{b}, Z_{d}\right)
$$


[see Theorem 2.3.2 in Brillinger (1981)] we can split $B_{T}\left(j_{1}, j_{2}\right)$ into two parts, and the independence of the innovations $Z_{t}$ yields for the first term

$$
\begin{aligned}
& \frac{1}{(2 \pi)^{2} N^{3}} \sum_{k_{1}, k_{2}=1}^{N / 2} \phi_{y_{1}, T}\left(j_{1}, \lambda_{k_{1}}\right) \phi_{y_{2}, T}\left(j_{2}, \lambda_{k_{2}}\right) \sum_{l, m, n, o=0}^{\infty} \psi_{l} \psi_{m} \psi_{n} \psi_{o} \\
& \times \sum_{\substack{p_{1}, p_{2}=0 \\
0 \leq p_{1}-l+o+j_{1}-j_{2} \leq N-1 \\
0 \leq p_{2}-n+m+j_{2}-j_{1} \leq N-1}}^{N-1} e^{-i \lambda_{k_{1}}\left(p_{1}-p_{2}+n-m-j_{2}+j_{1}\right)} e^{-i \lambda_{k_{2}}\left(p_{2}-p_{1}+l-o-j_{1}+j_{2}\right)} \\
& =\frac{1}{(2 \pi)^{2} N^{3}} \sum_{k_{1}, k_{2}=1}^{N / 2} \phi_{y_{1}, T}\left(j_{1}, \lambda_{k_{1}}\right) \phi_{y_{2}, T}\left(j_{2}, \lambda_{k_{2}}\right) \sum_{l, m, n, o=0}^{\infty} \psi_{l} \psi_{m} \psi_{n} \psi_{o} e^{-i \lambda_{k_{1}}(n-m)} e^{-i \lambda_{k_{2}}(l-o)} \\
& \times \sum_{\substack{p_{1}, p_{2}=0 \\
0 \leq p_{1}-l+o+j_{1}-j_{2} \leq N-1 \\
0 \leq p_{2}-n+m+j_{2}-j_{1} \leq N-1}}^{N-1} e^{-i\left(\lambda_{k_{1}}-\lambda_{k_{2}}\right)\left(p_{1}-p_{2}-j_{2}+j_{1}\right)}=: V_{k_{1}=k_{2}}+V_{k_{1} \neq k_{2}},
\end{aligned}
$$

where $V_{k_{1}=k_{2}}$ and $V_{k_{1} \neq k_{2}}$ denote the summation of all terms with $k_{1}=k_{2}$ and $k_{1} \neq k_{2}$ respectively [note that the restrictions $p_{1}-l+o+j_{1}-j_{2}=q_{2}$ and $p_{2}-n+m+j_{2}-j_{1}=q_{1}$ follow by the independence of the innovations $Z_{t}$ ]. For the first term we obtain

$$
\begin{aligned}
V_{k_{1}=k_{2}}= & \frac{1}{(2 \pi)^{2} N^{3}} \sum_{k=1}^{N / 2} \phi_{y_{1}, T}\left(j_{1}, \lambda_{k}\right) \phi_{y_{2}, T}\left(j_{2}, \lambda_{k}\right) \sum_{l, m, n, o=0}^{\infty} \psi_{l} \psi_{m} \psi_{n} \psi_{o} e^{-i \lambda_{k}(n-m)} e^{-i \lambda_{k}(l-o)} \\
& \times \max \left(N-1-\left|j_{1}-j_{2}-l+o\right|, 0\right) \max \left(N-1-\left|j_{2}-j_{1}+m-n\right|, 0\right) .
\end{aligned}
$$

By applying the summability condition (3.4) we obtain that $V_{k_{1}=k_{2}}$ is of order $O\left(\frac{1}{N}\right)$ if $\left|j_{1}-j_{2}\right|>N$, and that for $\Delta:=\left|j_{1}-j_{2}\right|<N$, 8.3) is the same as

$$
\frac{1}{N}\left(1-\frac{\Delta}{N}\right)^{2} \sum_{k=1}^{N / 2} \phi_{y_{1}, T}\left(j_{1}, \lambda_{k}\right) \phi_{y_{2}, T}\left(j_{2}, \lambda_{k}\right) f^{2}\left(\lambda_{k}\right)+O\left(\frac{1}{N}\right) .
$$


For the quantity $V_{k_{1} \neq k_{2}}$ we get by simple calculations and an application of (3.4)

$$
\begin{aligned}
V_{k_{1} \neq k_{2}}= & \frac{1}{(2 \pi)^{2} N^{3}} \sum_{\substack{k_{1}, k_{2}=1 \\
k_{1} \neq k_{2}}}^{N / 2} \phi_{y_{1}, T}\left(j_{1}, \lambda_{k_{1}}\right) \phi_{y_{2}, T}\left(j_{2}, \lambda_{k_{2}}\right) \sum_{l, m, n, o=0}^{\infty} \psi_{l} \psi_{m} \psi_{n} \psi_{o} e^{-i \lambda_{k_{1}}(n-m)} e^{-i \lambda_{k_{2}}(l-o)} \\
& \times \sum_{\substack{p_{1}, p_{2}=0 \\
0 \leq p_{1}+j_{1}-j_{2} \leq N-1 \\
0 \leq p_{2}+j_{2}-j_{1} \leq N-1}}^{N-1} e^{-i\left(\lambda_{k_{1}}-\lambda_{k_{2}}\right)\left(p_{1}-p_{2}-j_{2}+j_{1}\right)}+O\left(\frac{1}{N}\right)
\end{aligned}
$$

[i.e. we can omit the $l, m, n, o$ in the restrictions on $p_{i}$ at the cost of a term of order $O\left(\frac{1}{N}\right)$ ].

In the following let $a_{T}$ denote a sequence satisfying $a_{T} \rightarrow \infty, a_{T} / N \rightarrow 0$ and $N^{2} / a_{T}^{3} \rightarrow 0$.

It is straightforward to verify

$\sum_{\substack{p_{1}, p_{2}=0 \\ 0 \leq p_{1}+j_{1}-j_{2} \leq N-1 \\ 0 \leq p_{2}+j_{2}-j_{1} \leq N-1}}^{N-1} e^{-i\left(\lambda_{k_{1}}-\lambda_{k_{2}}\right)\left(p_{1}-p_{2}-j_{2}+j_{1}\right)}=\left|\sum_{p=0}^{N-1-\Delta} e^{-i\left(\lambda_{k_{1}}-\lambda_{k_{2}}\right) p}\right|^{2}=\left|\frac{1-e^{i \frac{2 \pi\left(k_{1}-k_{2}\right)}{N} \Delta}}{1-e^{-i \frac{2 \pi\left(k_{1}-k_{2}\right)}{N}}}\right|^{2}=\left|\frac{\sin \left(\frac{\pi\left(k_{1}-k_{2}\right)}{N} \Delta\right)}{\sin \left(\frac{\pi\left(k_{1}-k_{2}\right)}{N}\right)}\right|^{2}$

which yields

$$
\begin{aligned}
& V_{k_{1} \neq k_{2}}=\frac{1}{N^{3}} \sum_{\substack{k_{1}, k_{2}=1 \\
k_{1} \neq k_{2}}}^{N / 2} \phi_{y_{1}, T}\left(j_{1}, \lambda_{k_{1}}\right) \phi_{y_{2}, T}\left(j_{2}, \lambda_{k_{2}}\right) f\left(\lambda_{k_{1}}\right) f\left(\lambda_{k_{2}}\right)\left|\frac{\sin \left(\frac{\pi\left(k_{1}-k_{2}\right)}{N} \Delta\right)}{\sin \left(\frac{\pi\left(k_{1}-k_{2}\right)}{N}\right)}\right|^{2}+O\left(\frac{1}{N}\right) \\
& =\frac{1}{N^{3}} \sum_{\substack{k_{1}, k_{2}=1 \\
k_{1} \neq k_{2} \\
k_{1}-k_{2} \mid \leq a_{T}}}^{N / 2} \phi_{y_{1}, T}\left(j_{1}, \lambda_{k_{1}}\right) \phi_{y_{2}, T}\left(j_{2}, \lambda_{k_{2}}\right) f\left(\lambda_{k_{1}}\right) f\left(\lambda_{k_{2}}\right)\left|\frac{\sin \left(\frac{\pi\left(k_{1}-k_{2}\right)}{N} \Delta\right)}{\sin \left(\frac{\pi\left(k_{1}-k_{2}\right)}{N}\right)}\right|^{2}+O\left(\frac{1}{N}+\frac{1}{\left.a_{T}^{1-\tau}\right)}\right.
\end{aligned}
$$

for any $\tau>0$, because of

$$
\frac{1}{N} \sum_{\substack{k_{1}, k_{2}=1 \\\left|k_{1}-k_{2}\right|>a_{T}}}^{N / 2} \frac{1}{N^{2}} \frac{1}{\sin ^{2}\left(\frac{\pi\left(k_{1}-k_{2}\right)}{N}\right)} \leq \frac{C}{N} \sum_{\substack{k_{1}, k_{2}=1 \\\left|k_{1}-k_{2}\right|>a_{T}}}^{N / 2} \frac{1}{\left(k_{1}-k_{2}\right)^{2}} \leq \frac{1}{a_{T}^{1-\tau}}
$$


By applying $\sin (x)=x+O\left(\varepsilon^{3}\right)$ for $x \in[0, \varepsilon]$, a Taylor expansion and $\sum_{k=1}^{n} 1 / k \sim \log (n)$ we obtain, that $V_{k_{1} \neq k_{2}}$ is the same as

$$
\frac{1}{N} \sum_{\substack{k_{1}, k_{2}=1 \\ k_{1} \neq k_{2} \\\left|k_{1}-k_{2}\right| \leq a_{T}}}^{N / 2} \phi_{y_{1}, T}\left(j_{1}, \lambda_{k_{1}}\right) \phi_{y_{2}, T}\left(j_{2}, \lambda_{k_{1}}\right) f^{2}\left(\lambda_{k_{1}}\right)\left|\frac{\sin \left(\frac{\pi\left(k_{1}-k_{2}\right)}{N} \Delta\right)}{\pi\left(k_{1}-k_{2}\right)}\right|^{2}+O\left(\frac{1}{N}+\frac{1}{a_{T}^{1-\tau}}+\frac{N^{2}}{a_{T}^{3}}\right) .
$$

This expression is equal to

$$
\begin{aligned}
& \frac{1}{N} \sum_{k_{1}=a_{T}}^{N / 2-a_{T}} \phi_{y_{1}, T}\left(j_{1}, \lambda_{k_{1}}\right) \phi_{y_{2}, T}\left(j_{2}, \lambda_{k_{1}}\right) f^{2}\left(\lambda_{k_{1}}\right) \sum_{\substack{k_{2}=1 \\
k_{1} \neq k_{2} \\
\left|k_{1}-k_{2}\right| \leq a_{T}}}^{N / 2}\left|\frac{\sin \left(\frac{\pi\left(k_{1}-k_{2}\right)}{N}\right)}{\pi\left(k_{1}-k_{2}\right)}\right|^{2} \\
+ & O\left(\frac{1}{a_{T}^{1-\tau}}+\frac{a_{T}}{N}+\frac{N^{2}}{a_{T}^{3}}\right) \\
= & \frac{2}{N} \sum_{k_{1}=a_{T}}^{N / 2-a_{T}} \phi_{y_{1}, T}\left(j_{1}, \lambda_{k_{1}}\right) \phi_{y_{2}, T}\left(j_{2}, \lambda_{k_{1}}\right) f^{2}\left(\lambda_{k_{1}}\right)\left(\sum_{k_{2}=1}^{a_{T}} \frac{\sin ^{2}\left(\pi k_{2} \frac{\Delta}{N}\right)}{\pi^{2} k_{2}^{2}}\right)+O\left(\frac{1}{a_{T}^{1-\tau}}+\frac{a_{T}}{N}+\frac{N^{2}}{a_{T}^{3}}\right) \\
= & \frac{1}{N} \sum_{k_{1}=1}^{N / 2} \phi_{y_{1}, T}\left(j_{1}, \lambda_{k_{1}}\right) \phi_{y_{2}, T}\left(j_{2}, \lambda_{k_{1}}\right) f^{2}\left(\lambda_{k_{1}}\right) \frac{(N-\Delta) \Delta}{N^{2}}+O\left(\frac{1}{a_{T}^{1-\tau}}+\frac{a_{T}}{N}+\frac{N^{2}}{a_{T}^{3}}\right),
\end{aligned}
$$

where we used the identities $\sin ^{2}(x)=\frac{1}{2}(1-\cos (2 x))$ and $\sum_{k=1}^{\infty} \frac{\cos (k x)}{k^{2}}=\left(\frac{x-\pi}{2}\right)^{2}-\frac{\pi^{2}}{12}$ in the last step [see Jolley (1961)]. By combining the above results for $V_{k_{1}=k_{2}}$ and $V_{k_{1} \neq k_{2}}$ and proceeding completely analogously in the treatment of the second summand of $B_{T}\left(j_{1}, j_{2}\right)$ we obtain

$$
B_{T}\left(j_{1}, j_{2}\right)=\left(1-\frac{\Delta}{N}\right) \frac{2}{N} \sum_{k=1}^{N / 2} \phi_{y_{1}, T}\left(j_{1}, \lambda_{k}\right) \phi_{y_{2}, T}\left(j_{2}, \lambda_{k}\right) f^{2}\left(\lambda_{k}\right)+O\left(\frac{1}{a_{T}^{1-\tau}}+\frac{a_{T}}{N}+\frac{N^{2}}{a_{T}^{3}}\right)
$$


uniformly with respect to $\left(j_{1}, j_{2}\right)$ if $\Delta=\left|j_{1}-j_{2}\right|<N$. For the case $\frac{1}{c} \leq v_{i} \leq 1-\frac{1}{c}$ for $i=1,2$ the definition of the functions $\phi_{y_{1}, T}$ and $\phi_{y_{2}, T}$ now yields

$$
\begin{aligned}
& N \operatorname{Cov}\left(\hat{D}_{T}\left(\phi_{y_{1}, T}\right), \hat{D}_{T}\left(\phi_{y_{2}, T}\right)\right)=\sum_{\substack{\left(j_{1}, j_{2}\right) \in A_{T}\left(v_{1}, v_{2}\right) \\
\Delta<N}} B_{T}\left(j_{1}, j_{2}\right)+O\left(\frac{1}{a_{T}^{1-\varepsilon}}+\frac{a_{T}}{N}+\frac{N^{2}}{a_{T}^{3}}\right) \\
& \rightarrow \begin{cases}0 & \text { if } \frac{2}{c} \leq v_{2}-v_{1} \\
-\left[2-\left(v_{2}-v_{1}\right) c\right] \frac{1}{\pi} \int_{0}^{\min \left(\omega_{1}, \omega_{2}\right) \pi} f^{2}(\lambda) d \lambda & \text { if } \frac{1}{c}<v_{2}-v_{1} \leq \frac{2}{c} \\
{\left[2-3\left(v_{2}-v_{1}\right) c\right] \frac{1}{\pi} \int_{0}^{\min \left(\omega_{1}, \omega_{2}\right) \pi} f^{2}(\lambda) d \lambda} & \text { if } 0 \leq v_{2}-v_{1} \leq \frac{1}{c},\end{cases}
\end{aligned}
$$

and the assertion for the cases $v_{i}<\frac{1}{c}$ and $v_{i}>1-\frac{1}{c}$ for at least one $i \in\{1,2\}$ follows by similar arguments.

For the proof of part (iii) we use the notation $Y_{i, 1}:=Z_{j_{i}-N / 2+p_{i}-m_{i}}, Y_{i, 2}:=Z_{j_{i}-N / 2+q_{i}-n_{i}}$,

$$
A_{T, l}\left(v_{1}, \ldots, v_{l}\right):=\left\{\left\lfloor v_{1} T\right\rfloor-N / 2,\left\lfloor v_{1} T\right\rfloor+N / 2\right\} \times \ldots \times\left\{\left\lfloor v_{l} T\right\rfloor-N / 2,\left\lfloor v_{l} T\right\rfloor+N / 2\right\}
$$

and obtain as in the proof of Theorem 2.1 (2) in Preuß et al. (2012) that

$$
\operatorname{cum}\left(\sqrt{N} \hat{D}_{T}\left(\phi_{y_{1}, T}\right), \ldots, \sqrt{N} \hat{D}_{T}\left(\phi_{y_{l}, T}\right)\right)=\sum_{\nu} V(\nu)
$$

where $V(\nu)$ is defined by

$$
\begin{aligned}
& V(\nu):=\frac{1}{N^{\frac{3 l}{2}}(2 \pi)^{l}} \sum_{\left(j_{1}, \ldots, j_{l}\right) \in A_{T, l}\left(v_{1}, \ldots, v_{l}\right)} \sum_{k_{1}, \ldots, k_{l}=1}^{N / 2} \sum_{m_{1}, \ldots, m_{l}=0}^{\infty} \sum_{n_{1}, \ldots, n_{l}=0}^{\infty} \sum_{p_{1}, \ldots, p_{l}, q_{1}, \ldots, q_{l}=0}^{N-1} \\
& \quad \times \prod_{s=1}^{l} \phi_{y_{s}, T}\left(j_{s}, \lambda_{k_{s}}\right) \psi_{m_{s}} \psi_{n_{s}} e^{-\lambda_{k_{s}}\left(p_{s}-q_{s}\right)} \operatorname{cum}\left(Y_{a, b} ;(a, b) \in \nu_{1}\right) \cdots \operatorname{cum}\left(Y_{a, b} ;(a, b) \in \nu_{l}\right),
\end{aligned}
$$

and the summation is carried out over all indecomposable partitions $\nu=\left(\nu_{1}, \ldots, \nu_{k}\right)$ of the 
table

$$
\begin{array}{cc}
Y_{1,1} & Y_{1,2} \\
\vdots & \vdots \\
Y_{l, 1} & Y_{l, 2}
\end{array}
$$

[see Theorem 2.3.2 in Brillinger (1981)]. Due to the Gaussianity of the $Y_{i, j}$ we only have to consider partitions $\nu=\left(\nu_{1}, \ldots, \nu_{l}\right)$ with $l$ elements and without loss of generality we restrict ourselves to the indecomposable partition

$$
\bar{\nu}:=\bigcup_{i=1}^{l-1}\left(Y_{i, 1}, Y_{i+1,2}\right) \cup\left(Y_{l, 1}, Y_{1,2}\right)
$$

[all partitions which yield cumulants of the same order can be treated in the same way, while all other partitions yield cumulants of smaller order]. Simple calculations reveal

$$
\begin{aligned}
V(\bar{\nu}) & =\frac{1}{N^{\frac{3}{2}} l(2 \pi)^{l}} \sum_{\left(j_{1}, \ldots, j_{l}\right) \in A_{T, l}\left(v_{1}, \ldots, v_{l}\right)} \sum_{k_{1}, \ldots, k_{l}=1}^{N / 2} \prod_{s=1}^{l} \phi_{y_{s}, T}\left(j_{s}, \lambda_{k_{s}}\right) \sum_{m_{1}, \ldots, m_{l}, n_{1}, \ldots, n_{l}=0}^{\infty} \sum_{p_{1}, \ldots, p_{l}}^{\star} \\
& \times \prod_{s=1}^{l} \psi_{m_{s}} \psi_{n_{s}} e^{-i \lambda_{k_{1}}\left(p_{1}-p_{l}+m_{l}-n_{1}+j_{1}-j_{l}\right)} \prod_{s=2}^{l} e^{-i \lambda_{k_{s}}\left(p_{s}-p_{s-1}+m_{s-1}-n_{s}-j_{s-1}+j_{s}\right)}
\end{aligned}
$$

where $\sum^{\star}$ denotes the summation with respect to the conditions

$$
\begin{aligned}
0 & \leq q_{i+1}=p_{i}-m_{i}+n_{i+1}+j_{i}-j_{i+1} \leq N-1 \quad \text { for } \quad i \in\{1, \ldots, l-1\} \\
& 0 \leq q_{1}=p_{l}-m_{l}+n_{1}-j_{1}+j_{l} \leq N-1,
\end{aligned}
$$

[otherwise the cumulant vanishes due to the independence of the $Z_{t}$. (8.7) implies that

$$
\begin{aligned}
& \left|n_{i+1}-m_{i}+j_{i}-j_{i+1}\right| \leq N \quad \text { for } \quad i \in\{1, \ldots, l-1\} \\
& \left|n_{1}-m_{l}-j_{1}+j_{l}\right| \leq N
\end{aligned}
$$


have to hold for each summand in 8.6 not to vanish. If we combine this with the inequality

$$
\frac{1}{N} \sum_{k=1}^{N / 2} \phi_{y, T}\left(j, \lambda_{k}\right) e^{-i \lambda_{k} r} \leq \frac{C}{|r \bmod N / 2|}
$$

which holds uniformly with respect to $v, \omega$ and for all $r \in \mathbb{N}$ with $r \bmod N / 2 \neq 0$ [see (A2) in Eichler (2008)], and the bound $\psi_{l} \leq C l^{-1}$, which follows from (3.4), we obtain that (8.6) is bounded by (up to a constant)

$$
\begin{aligned}
& \frac{1}{N^{l / 2}} \sum_{\left(j_{1}, \ldots, j_{l}\right) \in A_{T, l}\left(v_{1}, \ldots, v_{l}\right)} \sum_{\substack{m_{1} \ldots n_{l}=1 \\
\left|n_{i+1}-m_{i}+j_{i}-j_{i+1} \leq N\\
\right| n_{1}-m_{l}-j_{1}+j_{l} \leq N}}^{\infty} \frac{1}{m_{1} \ldots m_{l} \cdot n_{1} \ldots n_{l}} \sum_{\substack{p_{1}, \ldots, p_{l}=0 \\
\left|p_{s}-p_{s-1}+m_{s}-1-n_{s}-j_{s-1}+j_{s}\right|<N / 2 \\
\left|p_{1}-p_{l}+m_{l}-n_{1}+j_{1}-j_{l}\right|<N / 2}}^{N-1} \\
& \times \prod_{s=1}^{l} \frac{1}{\left|p_{s}-p_{s-1}+m_{s-1}-n_{s}-j_{s-1}+j_{s}\right|} \prod_{s=1}^{l} 1\left(p_{s} \notin\left\{z_{s 1}, z_{s 2}\right\}\right),
\end{aligned}
$$

where we define $z_{s 1}:=-p_{s-1}+m_{s-1}-n_{s}-j_{s-1}+j_{s}$ and $z_{s 2}:=p_{s+1}+m_{s}-n_{s+1}-j_{s}+j_{s+1}$ (identifying 0 with $l$ and $l+1$ with 1 ) and use that the cases with $p_{s}=z_{s 1}, p_{s}=z_{s 2}$ or $\left|p_{s}-p_{s-1}+m_{s-1}-n_{s}-j_{s-1}+j_{s}\right| \geq N / 2$ for some $s \in\{1, \ldots, l\}$ are of the same or smaller order than (8.9). By proceeding completely analogously to the proof of Theorem 6.1 c) in Preuß and Vetter (2012) [with $d=0$ ] thereafter, it follows that $V(\bar{\nu})$ is of order $o(1)$.

\subsection{Proof of $(7.10)$ and $(7.11)$ in the proof of Theorem 3.1}

Since the proof of 7.10 is elementary we restrict ourselves to a treatment of (7.11), and by following the proof of Theorem 2.4 in Dahlhaus (1988), it suffices to show

$$
\left|\operatorname{cum}_{l}\left(\sqrt{N}\left(\hat{D}_{T}^{(1)}\left(y_{1}\right)-\hat{D}_{T}^{(1)}\left(y_{2}\right)\right)\right)\right| \leq(2 l) ! C^{l} d_{T}\left(y_{1}, y_{2}\right)^{l}
$$

for all $\left(y_{1}, y_{2}\right) \in \mathcal{P}_{T}, l \in \mathbb{N}$. Without loss of generality we assume that $l$ is even and $\omega_{1} \leq \omega_{2}$.

We use the notations $\phi(j, \lambda):=\phi_{y_{1}, T}^{(1)}(j, \lambda)-\phi_{y_{2}, T}^{(1)}(j, \lambda)$ and obtain as in the proof of part $(1)$ 
(iii) that

$$
\operatorname{cum}_{l}\left(\sqrt{N}\left(\hat{D}_{T}^{(1)}\left(y_{1}\right)-\hat{D}_{T}^{(1)}\left(y_{2}\right)\right)\right)=\sum_{\nu} V(\nu)
$$

where $V(\nu)$ is defined as

$$
\begin{aligned}
V(\nu) & :=\frac{1}{N^{\frac{3 l}{2}}(2 \pi)^{l}} \sum_{j_{1}, \ldots, j_{l}=1}^{T} \sum_{k_{1}, \ldots, k_{l}=1}^{N / 2} \sum_{m_{1}, \ldots, m_{l}=0}^{\infty} \sum_{n_{1}, \ldots, n_{l}=0}^{\infty} \sum_{p_{1}, \ldots, p_{l}, q_{1}, \ldots, q_{l}=0}^{N-1} \\
& \times \prod_{s=1}^{l} \phi\left(j_{s}, \lambda_{k_{s}}\right) \psi_{m_{s}} \psi_{n_{s}} e^{-\lambda_{k_{s}}\left(p_{s}-q_{s}\right)} \operatorname{cum}\left(Y_{a, b} ;(a, b) \in \nu_{1}\right) \cdots \operatorname{cum}\left(Y_{a, b} ;(a, b) \in \nu_{l}\right),
\end{aligned}
$$

and the summation is carried out over all indecomposable partitions $\nu=\left(\nu_{1}, \ldots, \nu_{k}\right)$ of (8.4). As in the proof of part (1) (iii), we only have to consider partitions $\nu=\left(\nu_{1}, \ldots, \nu_{l}\right)$ with $l$ elements due to the Gaussianity of the innovations and restrict ourselves to the indecomposable partition of the form (8.5). The definition of the function $\phi(j, \lambda)$ implies that the summation over the set $\{1, \ldots, T\}^{l}$ in 8.11 needs only to be performed over the set

$$
A_{T}\left(v_{1}, v_{2}\right)^{l}:=\left\{\left\lfloor v_{1} T\right\rfloor+N / 2,\left\lfloor v_{2} T\right\rfloor+N / 2\right\}^{l} .
$$

By additionally exploiting that $\operatorname{cum}\left(Z_{i}, Z_{j}\right)=\delta_{i j}$, we again obtain the $l$ relations 8.7) between $p_{i}, q_{i}, m_{i}, n_{i}$ and $j_{i}$, which have to be fulfilled such that the corresponding summands in 8.11 do not vanish. We redefine the $m_{i}$ and $n_{i}$ [denoting $m_{i}$ by $n_{i}$ and replacing $n_{i}$ by $\left.m_{i-1}\right]$, which gives the following conditions

$$
\begin{aligned}
& 0 \leq q_{i+1}=p_{i}+m_{i}-n_{i}+j_{i}-j_{i+1} \leq N-1 \text { for } i \in\{1,3, \ldots, l-1\} \\
& 0 \leq q_{i+1}=p_{i}+m_{i}-n_{i}+j_{i}-j_{i+1} \leq N-1 \text { for } i \in\{2,4, \ldots, l-2\} \\
& 0 \leq q_{1}=p_{l}+m_{l}-n_{l}+j_{l}-j_{1} \leq N-1 .
\end{aligned}
$$


By employing the Cauchy Schwarz inequality we can bound $V(\bar{\nu})$ by $\sqrt{V_{1}(\bar{\nu}) V_{2}(\bar{\nu})}$, where

$$
\begin{aligned}
& V_{1}(\bar{\nu}):=\frac{1}{N^{\frac{3 l}{2}}(2 \pi)^{l}} \sum_{\left(j_{1}, \ldots, j_{l}\right) \in A_{T}\left(v_{1}, v_{2}\right)^{l}} \sum_{k_{1}, \ldots, k_{l}=1}^{N / 2}\left(\prod_{s=1}^{l} \phi\left(j_{s}, \lambda_{k_{s}}\right)\right) \mid \sum_{p_{1}, p_{3} \ldots, p_{l-1}=0}^{N-1} \sum_{\substack{m_{1}, n_{1}, m_{3}, n_{3}, \ldots \\
, m_{l-1}, n_{l-1}=0}}^{\infty} \\
& \times\left.\prod_{s \in\{1,3, \ldots, l-1\}} \psi_{m_{s}} \psi_{n_{s}} \prod_{s \in\{1,3, \ldots, l-1\}} e^{-i\left(\lambda_{k_{s}}-\lambda_{k_{s+1}}\right) p_{s}} \prod_{s \in\{1,3, \ldots, l-1\}} e^{-i \lambda_{k_{s+1}}\left(n_{s}-m_{s}+j_{s+1}-j_{s}\right)}\right|^{2}
\end{aligned}
$$

and $V_{2}(\bar{\nu})$ is defined analogously with the respective summations carried out over $p_{i}, m_{i}, n_{i}$ for even $i$ and with respect to conditions (8.13) and (8.14). The term $V_{1}(\bar{\nu})$ is bounded by

$$
\frac{C}{N^{3 l / 2}} \sum_{\left(j_{1}, \ldots, j_{l}\right) \in A_{T}\left(v_{1}, v_{2}\right)^{l}} \sum_{k_{1}, k_{2} \ldots, k_{l}=1}^{N / 2}\left(\prod_{s=1}^{l} \phi\left(j_{s}, \lambda_{k_{s}}\right)\right)\left|H_{T}\left(j_{1}, j_{2}, \ldots, j_{l}, \lambda_{k_{1}}, \lambda_{k_{2}}, \ldots, \lambda_{k_{l}}\right)\right|
$$

where $H_{T}\left(j_{1}, j_{2}, \ldots, j_{l}, \lambda_{k_{1}}, \lambda_{k_{2}}, \ldots, \lambda_{k_{l}}\right)$ equals

$$
\left.\times e^{-i \lambda_{k_{s+1}}\left(-p_{s}+\bar{p}_{s}+n_{s}-m_{s}-\bar{n}_{s}+\bar{m}_{s}\right)}\right)
$$


and 8.12 denotes the condition 8.12 with $p_{i}, m_{i}, n_{i}$ replaced by $\bar{p}_{i}, \bar{m}_{i}, \bar{n}_{i}$. Simple calculations show that 8.16 is equal to

$$
\begin{aligned}
& \frac{C}{N^{3 l / 2}} \sum_{\left(j_{1}, \ldots, j_{l}\right) \in A_{T}\left(v_{1}, v_{2}\right)^{l}} \sum_{k_{1}, k_{2} \ldots, k_{l}=1}^{N / 2}\left(\prod_{s=1}^{l} \phi\left(j_{s}, \lambda_{k_{s}}\right)\right) \sum_{\substack{m_{1}, n_{1}, m_{3}, n_{3}, \ldots, \ldots \\
\ldots, m_{l}, 1, n_{l-1}=0}}^{\infty} \sum_{\substack{\bar{m}_{1}, \bar{n}_{1}, \bar{m}_{3}, \bar{n}_{3}, \ldots \\
\bar{m}_{l-1}, \bar{n}_{l-1}=0}}^{\infty} \\
& \times \prod_{s \in\{1,3, \ldots, l-1\}} \psi_{m_{s}} \psi_{n_{s}} \psi_{\bar{m}_{s}} \psi_{\bar{n}_{s}} \prod_{s \in\{2,4, \ldots, l\}} e^{-i \lambda_{k_{s}}\left(n_{s-1}-m_{s-1}-\bar{n}_{s-1}+\bar{m}_{s-1}\right)} \sum_{p_{1}, p_{3} \ldots, p_{l-1}=0}^{N-1} \sum_{\bar{p}_{1}, \bar{p}_{3} \ldots, \bar{p}_{l-1}=0}^{N-1} \\
& \times \prod_{s \in\{1,3, \ldots, l-1\}} e^{-i\left(\lambda_{k_{s}}-\lambda_{k_{s+1}}\right)\left(p_{s}-\bar{p}_{s}\right)} \\
& =\left(\frac{C}{N^{3}} \sum_{\left(j_{1}, j_{2}\right) \in A_{T}\left(v_{1}, v_{2}\right)^{2}} \sum_{k_{1}, k_{2}=1}^{N / 2} \phi\left(j_{1}, \lambda_{k_{1}}\right) \phi\left(j_{2}, \lambda_{k_{2}}\right) \sum_{m, n, \bar{m}, \bar{n}=0}^{\infty} \psi_{m} \psi_{n} \psi_{\bar{m}} \psi_{\bar{n}} e^{-i \lambda_{k_{2}}(n-m-\bar{n}+\bar{m})}\right. \\
& \left.\times \sum_{\substack{p_{1}, \bar{p}_{1}=0 \\
0 \leq p_{1}+m_{1}-n_{1}+j_{1}-j_{2} \leq N_{1} \\
0 \leq \bar{p}_{1}+\bar{m}_{1}-\bar{n}_{1}+j_{1}-j_{2} \leq N_{1}}}^{N-1} e^{-i\left(\lambda_{k_{1}}-\lambda_{k_{2}}\right)\left(p_{1}-\bar{p}_{1}\right)}\right)^{l / 2}=:\left(\sum_{\left(j_{1}, j_{2}\right) \in A_{T}\left(v_{1}, v_{2}\right)^{2}} \tilde{B}_{T}\left(j_{1}, j_{2}\right)\right)^{l / 2},
\end{aligned}
$$

where the quantities $\tilde{B}_{T}\left(j_{1}, j_{2}\right)$ are defined in an obvious manner. By the same arguments as provided in the calculation of the covariances and by the definition of $\phi$ one obtains that $\tilde{B}_{T}\left(j_{1}, j_{2}\right)=0$ if $\left|j_{1}-j_{2}\right|>N$ and that 8.17 is bounded by

$$
C\left[\int_{\omega_{1} \pi}^{\omega_{2} \pi} f^{2}(\lambda) d \lambda+\frac{2\left\lfloor\left\lfloor u\left(v_{1}, T\right) T\right\rfloor-\left\lfloor u\left(v_{2}, T\right) T\right\rfloor \mid\right.}{N} \int_{0}^{\omega_{1} \pi} f^{2}(\lambda) d \lambda\right]^{l / 2} \leq C d_{T}\left(y_{1}, y_{2}\right)^{l / 2} .
$$

Since the same upper bound holds for $V_{2}(\bar{\nu})$, the claim follows.

\subsection{Proof of Theorem 3.2}

As in the proof of Theorem 3.1 we restrict ourselves to the case $d=1$ and emphazise that $C$ denotes a constant, which does not depend on the sample size and can vary from line to line in the calculations. 
Proof of part a): In order to show the claim it is sufficient to prove the following two properties [see. Newey (1991)]:

(1) For every $y:=(v, \omega) \in[0,1]$ we have

$$
N^{\gamma} \hat{D}_{T}(y)=o_{P}(1)
$$

(2) For every $\eta, \varepsilon>0$ there exists a $\delta>0$ such that

$$
\lim _{T \rightarrow \infty} P\left(\sup _{\left(y_{1}, y_{2}\right) \in[0,1]^{2}: d_{2}\left(y_{1}, y_{2}\right)<\delta} N^{\gamma}\left|\hat{D}_{T}\left(y_{1}\right)-\hat{D}_{T}\left(y_{2}\right)\right|>\eta\right)<\varepsilon,
$$

where $d_{2}\left(y_{1}, y_{2}\right)$ denotes the euclidean distance between $y_{1}=\left(v_{1}, \omega_{1}\right)$ and $y_{2}=\left(v_{2}, \omega_{2}\right)$.

The statement (8.18) follows by similar arguments as given in the proof of Theorem 3.1 a) and the details are omitted for the sake of brevity. For a proof of $(8.19)$ we employ the representation

$$
\hat{D}_{T}(y)=\frac{1}{N} \sum_{j=1}^{T} \sum_{k=1}^{N / 2} \phi_{y, T}\left(j, \lambda_{k}\right) I_{N}\left(\frac{j}{T}, \lambda_{k}\right)
$$

which was introduced in (7.4), where the functions $\phi_{y, T}$ for $y=(v, \omega)$ are defined in (7.3). We define by

$$
\rho_{2, T, \gamma}\left(y_{1}, y_{2}\right):=\left(\frac{1}{N^{2(1-\gamma)}} \sum_{j=1}^{T} \sum_{k=1}^{N}\left(\phi_{y_{1}, T}\left(j, \lambda_{k}\right)-\phi_{y_{2}, T}\left(j, \lambda_{k}\right)\right)^{2}\right)^{1 / 2}
$$

a semi-metric on the set

$$
\mathcal{P}_{T}:=\{N / T,(N+1) / T, \ldots, 1-N / T\} \times\{1 / N, \ldots, 1-1 / N, 1\},
$$


and a straightforward calculation shows that there exists a constant $A \in \mathbb{R}^{+}$such that the inequality

$$
\begin{aligned}
& \rho_{2, T, \gamma}\left(y_{1}, y_{2}\right) \leq d_{T, \gamma}\left(y_{1}, y_{2}\right) \\
& \quad:=\frac{A / 2}{N^{\frac{1}{2}-\gamma}}\left(\sqrt{\left|\omega_{2}-\omega_{1}\right|}+\sqrt{\left|v_{2}-v_{1}\right|}\right) 1_{\left\{\left\lfloor v_{1} T\right\rfloor=\left\lfloor v_{2} T\right\rfloor\right\}}+\frac{A}{N^{\frac{1}{2}-\gamma}} 1_{\left\{\left\lfloor v_{1} T\right\rfloor \neq\left\lfloor v_{2} T\right\rfloor\right\}}
\end{aligned}
$$

holds. Note that $d_{T, \gamma}(\cdot, \cdot)$ defines a further semi-metric on $\mathcal{P}_{T}$, and for every $\gamma \in[0,1 / 2)$ we obtain for fixed $\delta>0$ and sufficiently large $T \in \mathbb{N}$

$$
\mathbb{P}\left(\sup _{\substack{y_{i} \in[0,1]: \\ d_{2}\left(y_{1}, y_{2}\right)<\delta}} N^{\gamma}\left|\hat{D}_{T}\left(y_{1}\right)-\hat{D}_{T}\left(y_{2}\right)\right|>\eta\right) \leq \mathbb{P}\left(\sup _{\substack{y_{i} \in \mathcal{P}_{T} \\ d_{T, \gamma}\left(y_{1}, y_{2}\right)<\delta}} N^{\gamma}\left|\hat{D}_{T}\left(y_{1}\right)-\hat{D}_{T}\left(y_{2}\right)\right|>\eta\right)
$$

So it suffices to prove that the probability on the right hand side of $(8.22)$ is arbitrary small for $T$ sufficiently large. This is a consequence of the following assertions:

i) $\lim _{\kappa \rightarrow 0} \lim _{T \rightarrow \infty} J_{T, \gamma}(\kappa)=0$, where $J_{T, \gamma}$ denotes the covering integral of the set $\mathcal{P}_{T}$ with respect to the metric $d_{T, \gamma}$.

ii) There exists a constant $C \in \mathbb{R}^{+}$such that for all $y_{1}, y_{2} \in[0,1]^{2}$ and integers $l \in \mathbb{N}$

$$
\left|\operatorname{cum}_{l}\left(N^{\gamma}\left(\hat{D}_{T}\left(y_{1}\right)-\hat{D}_{T}\left(y_{2}\right)\right)\right)\right| \leq(2 l) ! C^{l} d_{T, \gamma}\left(y_{1}, y_{2}\right)^{k}
$$

Proof of part i): The construction of $d_{T, \gamma}$ implies that there exists a constant $A>0$ such that $C\left(u, d_{T, \gamma}, \mathcal{P}_{T}\right) \leq T N$ for $u \leq A / N^{1 / 2-\gamma}$ and $C\left(u, d_{T, \gamma}, \mathcal{P}_{T}\right)=1$ for $u \geq A / N^{1 / 2-\gamma}$. This yields that, for any $\kappa>0$, the quantity $J_{T, \gamma}(\kappa)$ is bounded by

$$
\begin{aligned}
& J_{T, \gamma}\left(A / N^{1 / 2-\gamma}\right)+1_{\left\{\kappa>A / N^{1 / 2-\gamma}\right\}} \int_{A / N^{1 / 2-\gamma}}^{\kappa}\left[\log \left(\frac{48 C\left(u, d_{T, \gamma}, \mathcal{P}_{T}\right)^{2}}{u}\right)\right]^{2} d u \\
\leq & \int_{0}^{A / N^{1 / 2-\gamma}}\left[\log \left(48 T^{2} N^{2}\right)^{2}-2 \log \left(48 T^{2} N^{2}\right) \log (u)+\log (u)^{2}\right] d u+\left|\int_{A / N^{1 / 2-\gamma}}^{\kappa}\left[\log \left(\frac{48}{u}\right)\right]^{2} d u\right|,
\end{aligned}
$$


and it is straightforward to see that the right handside of the above expression converges to zero as $T \rightarrow \infty$ and $\kappa \rightarrow 0$.

Proof of part ii): We assume without loss of generality that $l$ is even and only consider the first summand $\left\{\hat{D}_{T}^{(1)}(y)\right\}_{y \in[0,1]^{2}}$. We then obtain as in the proof of 7.11) (by using an additional symmetry argument) that

$$
\operatorname{cum}_{l}\left(N^{\gamma}\left(\hat{D}_{T}^{(1)}\left(y_{1}\right)-\hat{D}_{T}^{(1)}\left(y_{2}\right)\right)\right)=\sum_{\nu} V_{\gamma}(\nu)
$$

where $V_{\gamma}(\nu)$ is defined as

$$
\begin{aligned}
V_{\gamma}(\nu):= & \frac{1}{N^{l(2-\gamma)}(4 \pi)^{l}} \sum_{j_{1}, \ldots, j_{l}=1}^{T} \sum_{k_{1}, \ldots, k_{l}=-\left\lfloor\frac{N-1}{2}\right\rfloor}^{N / 2} \sum_{m_{1}, \ldots, m_{l}=0}^{\infty} \sum_{n_{1}, \ldots, n_{l}=0}^{\infty} \sum_{p_{1}, \ldots, p_{l}, q_{1}, \ldots, q_{l}=0}^{N-1} \prod_{s=1}^{l} \phi\left(j_{s}, \lambda_{k_{s}}\right) \\
& \times \prod_{s=1}^{l}\left[\psi_{m_{s}} \psi_{n_{s}} e^{-\lambda_{k_{s}}\left(p_{s}-q_{s}\right)}\right] \operatorname{cum}\left(Y_{a, b} ;(a, b) \in \nu_{1}\right) \cdots \operatorname{cum}\left(Y_{a, b} ;(a, b) \in \nu_{l}\right),
\end{aligned}
$$

and the summation is carried out over all indecomposable partitions $\nu=\left(\nu_{1}, \ldots, \nu_{k}\right)$ of 8.4$)$ and without loss of generality we restrict ourselves to the indecomposable partition defined in 8.5 . By proceeding completely analogously to the proof of (7.11) we obtain

$$
V_{\gamma}(\bar{\nu}) \leq \sqrt{V_{1, \gamma}(\bar{\nu}) V_{2, \gamma}(\bar{\nu})}
$$

where

$$
\begin{aligned}
V_{1, \gamma}(\bar{\nu}) & :=\frac{1}{N^{l(2-\gamma)(4 \pi)^{l}}} \sum_{\left(j_{1}, \ldots, j_{l}\right) \in A_{T}\left(v_{1}, v_{2}\right)^{l} l} \sum_{k_{1}, \ldots, k_{l}=-\left\lfloor\frac{N-1}{2}\right\rfloor}^{N / 2} \prod_{s \in\{1,3, \ldots, l-1\}} \phi^{2}\left(j_{s}, \lambda_{k_{s}}\right) \\
& \times \sum_{p_{1}, p_{3} \ldots, p_{l-1}=0}^{N-1} \prod_{m_{1}, n_{1}, m_{3}, n_{3}, \ldots, m_{l-1}, n_{l-1}=0}^{(8.12\}} \psi_{s \in\{1,3, \ldots, l-1\}} \psi_{m_{s}} \psi_{n_{s}} \prod_{s \in\{1,3, \ldots, l-1\}} e^{-i\left(\lambda_{k_{s}}-\lambda_{k_{s+1}}\right) p_{s}} \\
& \times\left.\prod_{s \in\{1,3, \ldots, l-1\}} e^{-i \lambda_{k_{s+1}}\left(n_{s}-m_{s}+j_{s+1}-j_{s}\right)}\right|^{2}
\end{aligned}
$$


and $V_{2, \gamma}(\bar{\nu})$ is defined analogously with the respective summations carried out over $j_{i}, p_{i}, m_{i}, n_{i}$ for even $i$ and with respect to condition 8.14 . The term $V_{1, \gamma}(\bar{\nu})$ is bounded by

$$
\frac{C}{N^{l(1-\gamma)}} \sum_{\substack{\left(j_{1}, \ldots, j_{l}\right) \\ \in A_{T}\left(v_{1}, v_{2}\right)^{l}}} \sum_{k_{1}, k_{3} \ldots, k_{l-1}=-\left\lfloor\frac{N-1}{2}\right\rfloor}^{N / 2} \prod_{s \in\{1,3, \ldots, l-1\}} \phi^{2}\left(j_{s}, \lambda_{k_{s}}\right)\left|H_{T, 2}\left(j_{1}, j_{2}, \ldots, j_{l}, \lambda_{k_{1}}, \lambda_{k_{3}}, \ldots, \lambda_{k_{l-1}}\right)\right|,
$$

where $H_{T, 2}\left(j_{1}, j_{2}, \ldots, j_{l}, \lambda_{k_{1}}, \lambda_{k_{3}}, \ldots, \lambda_{k_{l-1}}\right)$ equals

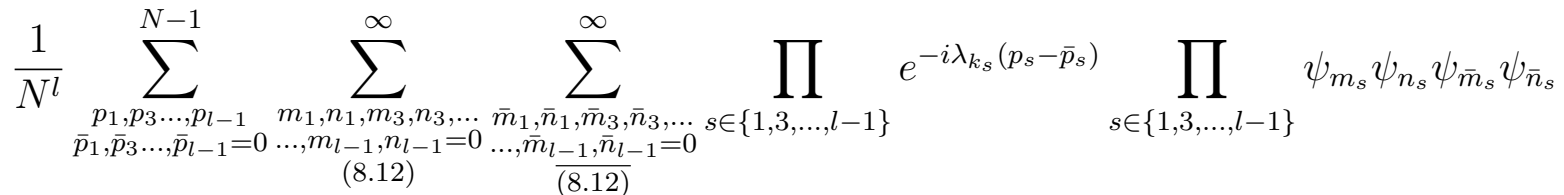

$$
\begin{aligned}
& \times \sum_{k_{2}, \ldots, k_{l}=-\left\lfloor\frac{N-1}{2}\right\rfloor}^{N / 2} \prod_{s \in\{1,3, \ldots, l-1\}} e^{-i \lambda_{k_{s+1}}\left(-p_{s}+\bar{p}_{s}+n_{s}-m_{s}-\bar{n}_{s}+\bar{m}_{s}\right)}
\end{aligned}
$$

and $\sqrt{8.12}$ denotes the condition $(8.12)$ with $p_{i}, m_{i}, n_{i}$ replaced by $\bar{p}_{i}, \bar{m}_{i}, \bar{n}_{i}$. The well know identity

$$
\frac{1}{N} \sum_{k=-\left\lfloor\frac{N-1}{2}\right\rfloor}^{N / 2} \exp \left(\lambda_{k} p\right)= \begin{cases}1 & \text { if } p=l N \text { for some } l \in \mathbb{N} \\ 0 & \text { else }\end{cases}
$$

yields that for a fixed choice of $p_{i}, m_{i}, n_{i}, \bar{m}_{i}, \bar{n}_{i}$ there is only one choice for $\bar{p}_{i}$ such that the corresponding summand does not vanish. Thus we obtain

$$
\begin{aligned}
& \sum_{\left(j_{2}, j_{4} \ldots, j_{l}\right) \in A_{T}\left(v_{1}, v_{2}\right)^{l / 2}}\left|H_{T, 2}\left(j_{1}, j_{2}, \ldots, j_{l}, \lambda_{k_{1}}, \lambda_{k_{3}}, \ldots, \lambda_{k_{l-1}}\right)\right| \\
\leq & \sum_{\left(j_{2}, j_{4} \ldots, j_{l}\right) \in A_{T}\left(v_{1}, v_{2}\right)^{l / 2}} \sum_{\substack{m_{1}, m_{3}, \ldots, m_{l-1} \\
\bar{m}_{1}, \bar{m}_{3} \ldots, \bar{m}_{l-1} \\
n_{1}, n_{3}, \ldots, n_{l-1} \\
\bar{n}_{1}, \bar{n}_{3}, \ldots, \bar{n}_{l-1}=0}}^{\infty} \prod_{s \in\{1,3, \ldots, l-1\}}\left|\psi_{m_{s}} \psi_{n_{s}} \psi_{\bar{m}_{s}} \psi_{\bar{n}_{s}}\right|=4^{l / 2}\left(\sum_{n=0}^{\infty}\left|\psi_{n}\right|\right)^{2 l},
\end{aligned}
$$


which is bounded by $C^{l}$ for some $C>0$ due to (3.4). Observing (8.25) this implies that $V_{1, \gamma}(\bar{\nu})$ is bounded by

$$
\frac{C^{l}}{N^{l(1-\gamma)}} \sum_{j_{1}, j_{3} \ldots, j_{l-1}=1}^{T} \sum_{k_{1}, k_{3} \ldots, k_{l-1}=-\left\lfloor\frac{N-1}{2}\right\rfloor}^{N / 2}\left(\phi^{2}\left(j_{1}, \lambda_{k_{1}}\right) \phi^{2}\left(j_{3}, \lambda_{k_{3}}\right) \ldots \phi^{2}\left(j_{l-1}, \lambda_{k_{l-1}}\right)\right)
$$

which equals $C^{l} \rho_{2, T, \gamma}\left(y_{1}, y_{2}\right)^{l}$. Since the same upper bound can be obtained for $V_{2}(\bar{\nu}),(8.23)$ follows with 8.21 .

Proof of part b): Assertion b) of Theorem 3.2 can be established using the same arguments as in the proof of part b) of Theorem 3.1 .

\subsection{Proof of Theorem 3.6}

We again consider only the case $d=1$ and suppress the argument $T$, when referring to the sequence $p=p(T)$. Note that due to Assumption 3.3 the stationary process $X_{t}$ with spectral density $g(\lambda)=\int_{0}^{1} f(u, \lambda) d u$ possesses an $\mathrm{MA}(\infty)$ representation

$$
X_{t}=\sum_{l=0}^{\infty} \psi_{l} Z_{t-l}
$$

Moreover, under the null hypothesis we have $g \equiv f$ and the functions $\psi_{l}(u)$ in (2.1) satisfy for all $u \in[0,1] \psi_{l}(u)=\psi_{l}(l \in \mathbb{N})$. Let $\left\{X_{t}^{*}\right\}_{t \in \mathbb{Z}}$ denote the process which is obtained by replacing $Z_{t}$ in (8.26) by the bootstrap replicates $Z_{t}^{*}$ from step 2) in Algorithm 3.4, then $\left\{X_{t}\right\}_{t \in \mathbb{Z}}$ and $\left\{X_{t}^{*}\right\}_{t \in \mathbb{Z}}$ obviously have the same distribution. Thus, if we define $\hat{D}_{T, a}^{*}(y)$ as $\hat{D}_{T}(y)$ where the random variables $X_{t, T}$ are replaced by $X_{t}^{*}$, then part a) of Theorem 3.6 follows directly.

From the arguments presented in the proof of Theorem 3.1 we obtain that there exist $\tilde{y} \in$ 
$[0,1]^{2}$ such that $\left|\hat{D}_{T, a}^{*}(\tilde{y})\right|^{2} \geq L / N$ for some constant $L \in \mathbb{R}^{+}$. So it suffices to prove

$$
\sqrt{N} \sup _{(y) \in[0,1]^{2}}\left|\hat{D}_{T}^{*}(y)-\hat{D}_{T, a}^{*}(y)\right|=o_{P}(1)
$$

which is a consequence of the following two statements [see the proof of Theorem 3.2]

(1) For every $y=(v, \omega) \in[0,1]$ we have $\sqrt{N}\left(\hat{D}_{T}^{*}(y)-\hat{D}_{T, a}^{*}(y)\right)=o_{P}(1)$.

(2) For every $\eta, \varepsilon>0$ there exists a $\delta>0$ such that

$$
\lim _{T \rightarrow \infty} P\left(\sup _{\substack{y_{1}, y_{2} \in[0,1)^{2}: \\ d_{2}\left(y_{1}, y_{2}\right)<\delta}} \sqrt{N}\left|\left(\hat{D}_{T}^{*}\left(y_{1}\right)-\hat{D}_{T, a}^{*}\left(y_{1}\right)\right)-\left(\hat{D}_{T}^{*}\left(y_{2}\right)-\hat{D}_{T, a}^{*}\left(y_{2}\right)\right)\right|>\eta\right)<\varepsilon .
$$

For the sake of brevity we restrict ourselves to a proof of (8.28) since it is by far the more complicated one. For this purpose we introduce the process

$$
X_{t}^{A R}(p):=\sum_{j=1}^{p} a_{j, p} X_{t-j}^{A R}(p)+Z_{t}^{A R}(p)
$$

where $a_{1, p}, \ldots, a_{p, p}$ are defined in (3.8) and the $Z_{j}^{A R}(p)$ are independent centered Gaussian random variables with variance $\sigma_{p}=\mathbb{E}\left[\left(X_{t}-\sum_{j=1}^{p} a_{j, p} X_{t-j}\right)^{2}\right]$. Because $\left\{X_{t}\right\}_{t \in \mathbb{Z}}$ is the stationary process with spectral density $g(\lambda)=\int_{0}^{1} f(u, \lambda) d u$, the process $\left\{X_{t}^{A R}(p)\right\}_{t \in \mathbb{Z}}$ corresponds to the 'best' approximation of the process (3.7) by an $\operatorname{AR}(p)$ model. It now follows from Lemma 2.3 in Kreiss et al. (2011) that for sufficiently large $T$ the approximating process $\left\{X_{t}^{A R}(p)\right\}_{t \in \mathbb{Z}}$ and the bootstrap analogon $X_{t, T}^{*}$ in $(3.9)$ have MA( $\left.\infty\right)$ representations, i.e.

$$
X_{t}^{A R}(p)=\sum_{j=0}^{\infty} \psi_{j}^{A R}(p) Z_{t-j}^{A R}(p) \quad \text { and } \quad X_{t, T}^{*}=\sum_{j=0}^{\infty} \hat{\psi}_{j}^{A R}(p) Z_{t-j}^{*}
$$

We define $W_{T}$ as the event

$$
\sqrt{N} \sum_{m, n=0}^{\infty}\left|\psi_{m} \psi_{n}-\hat{\psi}_{m}^{A R}(p) \hat{\psi}_{n}^{A R}(p)\right| \leq 1
$$


and $\bar{D}_{T}(y):=\sqrt{N}\left(\hat{D}_{T}^{*}(y)-\hat{D}_{T, a}^{*}(y)\right) \times 1_{W_{T}}$. The claim 8.28 then follows as in the proof of (8.19) from the following two assertions:

i) $\mathbb{P}\left(W_{T}\right) \rightarrow 1$.

ii) There exists a constant $C \in \mathbb{R}^{+}$such that for all $y_{1}=\left(v_{1}, \omega_{1}\right)$ and $y_{2}=\left(v_{2}, \omega_{2}\right)$

$$
\left|\operatorname{cum}_{l}\left(\bar{D}_{T}\left(y_{1}\right)-\bar{D}_{T}\left(y_{2}\right)\right)\right| \leq(2 l) ! C^{l} \rho_{2, T, 0}\left(y_{1}, y_{2}\right)^{l}, \quad \forall l \in \mathbb{N},
$$

where $\rho_{2, T, 0}$ was defined in 8.20 .

We will at first comment on the second statement 8.32 and justify the first part afterwards. If we define $V_{1, \gamma}(\bar{\nu})^{*}$ as $V_{1, \gamma}(\bar{\nu})$ in 8.15 , with the quantities

$$
\prod_{s \in\{1,3, \ldots, l-1\}} \psi_{m_{s}} \psi_{n_{s}}
$$

being replaced by

$$
\mathbb{E}\left(\prod_{s \in\{1,3, \ldots, l-1\}}\left(\psi_{m_{s}} \psi_{n_{s}}-\hat{\psi}_{m_{s}}^{A R} \hat{\psi}_{n_{s}}^{A R}\right)\right)
$$

then we obtain by the same arguments as given in the proof of Theorem 3.2 that $V_{1, \gamma}(\bar{\nu})^{*}$ is bounded by

$\frac{1}{(4 \pi)^{l} N^{l / 2}} \sum_{\substack{\left(j_{1}, \ldots, j_{l}\right) \\ \in A_{T}\left(v_{1}, v_{2}\right)}} \sum_{k_{1}, k_{3} \ldots, k_{l-1}=-\left\lfloor\frac{N-1}{2}\right\rfloor}^{N / 2} \prod_{s \in\{1,3, \ldots, l-1\}} \phi^{2}\left(j_{s}, \lambda_{k_{s}}\right)\left|H_{T, 2}^{*}\left(j_{1}, j_{2}, \ldots, j_{l}, \lambda_{k_{1}}, \lambda_{k_{3}}, \ldots, \lambda_{k_{l-1}}\right)\right|$,

where $H_{T, 2}^{*}\left(j_{1}, j_{2}, \ldots, j_{l}, \lambda_{k_{1}}, \lambda_{k_{3}}, \ldots, \lambda_{k_{l-1}}\right)$ is the corresponding bootstrap analogue of $H_{T, 2}\left(j_{1}, j_{2}, \ldots, j_{l}, \lambda_{k_{1}}, \lambda_{k_{3}}, \ldots, \lambda_{k_{l-1}}\right)$, i.e. the quantity (8.33) is replaced by 8.34). Combining 
the arguments in the treatment of $H_{T, 2}\left(j_{1}, \ldots, \lambda_{k_{l-1}}\right)$ with 8.31 we obtain

$$
\sum_{\left(j_{2}, j_{4}, \ldots, j_{l}\right) \in A_{T}\left(v_{1}, v_{2}\right)^{l / 2}} N^{l / 2}\left|H_{T, 2}^{*}\left(j_{1}, j_{2}, \ldots, j_{l}, \lambda_{k_{1}}, \lambda_{k_{3}}, \ldots, \lambda_{k_{l-1}}\right)\right| \leq 4^{l / 2}
$$

on the set $W_{T}$. This yields 8.32 as in the proof of 8.19$)$, and it remains to prove $\mathbb{P}\left(W_{T}\right) \rightarrow 1$.

Because of

$$
\sum_{m, n=0}^{\infty}\left|\psi_{m} \psi_{n}-\hat{\psi}_{m}^{A R}(p) \hat{\psi}_{n}^{A R}(p)\right| \leq \sum_{m=0}^{\infty}\left|\psi_{m}\right| \sum_{n=0}^{\infty}\left|\psi_{n}-\hat{\psi}_{n}^{A R}(p)\right|+\sum_{m=0}^{\infty}\left|\hat{\psi}_{m}^{A R}(p)\right| \sum_{n=0}^{\infty}\left|\psi_{n}-\hat{\psi}_{n}^{A R}(p)\right|
$$

it is sufficient to show

$$
\sqrt{N} \sum_{n=0}^{\infty}\left|\psi_{n}-\hat{\psi}_{n}^{A R}(p)\right|=o_{P}(1) \quad \text { and } \quad \sum_{m=0}^{\infty}\left|\hat{\psi}_{m}^{A R}(p)\right|=O_{P}(1)
$$

We now use Lemma 2.3 of Kreiss et al. (2011) which implies that the polynomials $A_{p}(z):=$ $1-\sum_{k=1}^{p} a_{j, p} z^{k}$ and $\hat{A}_{p}(z):=1-\sum_{k=1}^{p} \hat{a}_{j, p} z^{k}$ have no roots within the closed unit disc $\{z|| z \mid \leq$ $\left.1+\frac{1}{p}\right\}$ if $p$ is sufficiently large. An application of Cauchyï $₫ \frac{1}{2} \mathrm{~s}$ inequality for holomorphic functions to the difference

$$
\hat{A}_{p}^{-1}(z)-A_{p}^{-1}(z):=\sum_{k=1}^{\infty}\left[\hat{\psi}_{k}^{A R}(p)-\psi_{k}^{A R}(p)\right] z^{k}
$$

as in the proof of Lemma 2.5 in Kreiss et al. (2011), yields

$$
\begin{aligned}
\left|\hat{\psi}_{l}^{A R}(p)-\psi_{l}^{A R}(p)\right| & \leq\left(\frac{1}{1+1 / p}\right)^{l} \max _{|z|=1+\frac{1}{p}} \frac{\left|A_{p}(z)-\hat{A}_{p}(z)\right|}{\left|A_{p}(z) \hat{A}_{p}(z)\right|} \\
& \leq\left(\frac{1}{1+1 / p}\right)^{l} \max _{|z|=1+\frac{1}{p}} \frac{\sum_{k=1}^{p}\left|\hat{a}_{k, p}-a_{k, p}\right|\left(1+\frac{1}{p}\right)^{k}}{\left|A_{p}(z) \hat{A}_{p}(z)\right|} \\
& =p\left(1+\frac{1}{p}\right)^{-l} O_{P}(\sqrt{\log (T) / T})
\end{aligned}
$$


uniformly with respect to $l$ and $p$. This bound implies

$$
\sqrt{N} \sum_{n=0}^{\infty}\left|\hat{\psi}_{n}^{A R}(p)-\psi_{n}^{A R}(p)\right|=O_{P}\left(\frac{p_{\max }^{2}(T) \sqrt{\log (T)} \sqrt{N}}{\sqrt{T}}\right)=o_{P}(1)
$$

and Lemma 2.4 of Kreiss et al. (2011) yields that (for sufficiently large T)

$$
\sqrt{N} \sum_{n=0}^{\infty}\left|\psi_{n}^{A R}(p)-\psi_{n}\right| \leq \frac{\sqrt{N}}{p} \sum_{j=p+1}^{\infty} j\left|a_{j}\right|=O_{P}\left(\frac{\sqrt{N}}{p_{\min }(T)}\right)=o_{P}(1)
$$

(8.36) and (8.37) now imply the first part of 8.35). The second part can be easily derived from the first part and (3.4). This yields part b). Part c) is of course the bootstrap analogue of Theorem 3.2 a), and is therefore shown by combining the arguments given here with the reasoning in the proof of Theorem $3.2 \mathrm{a}$ ). 